\title{
On the many-time formulation of classical particle dynamics
}

\author{
G. Longhi \\ Dipartimento di Fisica, Universitá di Firenze, Firenze, Italy \\ L. Lusanna \\ Istituto Nazionale di Fisica Nucleare, Sezione di Firenze, Firenze, Italy
}

J. M. Pons

Departament de Fisica Teorica, Universitat de Barcelona, Barcelona, Spain

(Received 14 January 1988; accepted for publication 15 February 1989)

\begin{abstract}
Starting from the standard one-time dynamics of $n$ nonrelativistic particles, the $n$-time equations of motion are inferred, and a variational principle is formulated. A suitable generalization of the classical Lie-König theorem is demonstrated, which allows the determination of all the associated presymplectic structures. The conditions under which the action of an invariance group is canonical are studied, and a corresponding Noether theorem is deduced. A formulation of the theory in terms of $n$ first-class constraints is recovered by means of coisotropic imbeddings. The proposed approach also provides for a better understanding of the relativistic particle dynamics, since it shows that the different roles of the physical positions and the canonical variables is not peculiar to special relativity, but rather to any $n$-time approach: indeed a nonrelativistic no-interaction theorem is deduced.
\end{abstract}

\section{INTRODUCTION}

As it is well known, in the treatment of bound states in the framework of quantum field theory, both relativistic and nonrelativistic, the states of the bound system are described by a wave function for many particles, which will depend in a natural way on the times of each elementary field. In order to give a physical interpretation to this wave function, we must give a meaning to this many time description, or, what is the same, to have a consistent dynamical theory for systems of $n$ particles, with $n$ different times.

At the relativistic level the bound states are described by the Bethe-Salpeter equation, ' with the connected problems of the normalization and interpretation of its solutions. To get a better understanding of it, Todorov ${ }^{2}$ and then Komar ${ }^{3}$ developed a manifestly covariant classical relativistic model for two particles, of an action-at-a-distance kind, ${ }^{4}$ which describes in a covariant way the instantaneous approximations of the Bethe-Salpeter equation, restricted to the two particle sector. ${ }^{5}$ In the Todorov-Komar model the dynamics is given in terms of two first-class constraints, and, therefore, the relative time problem is related to the existence of gauge transformations generated by the constraints. ${ }^{6}$ An equivalent model was discovered by Droz-Vincent, ${ }^{7}$ which was based on a two-time formulation of the classical relativistic dynamics. Here we have the first example of the connection between the first-class constraints formulation and the many-time dynamics.

The Todorov-Komar-Droz-Vincent model for two particles, in its first quantized version, ${ }^{2,8-10}$ generates a bilocal wave function, which is a solution of two coupled integrable integro-differential wave equations. In Ref. 11 a complete analysis of these equations has been done, by giving the set of their solutions (where the relative time appears in a phase factor), the explicit expression of the Poincare invariant scalar products (see also Ref. 9 and 10 and the last paper quoted in Ref. 8), and, by solving the initial data problem, a probabilistic interpretation of the wave function is proposed. The connection with the Bethe-Salpeter equation is studied in Ref. 5, and, for the nonrelativistic limit, in Ref. 12. Attempts toward a second quantization along these lines are given in Ref. 13.

However, due to the complications introduced by special relativity, a clear understanding of all the involved structures, and a clear physical interpretation of them, is still lacking. One of these complications is for instance the problem of the most suitable definition of the relativistic position coordinates, see Ref. 14. The Todorov-Komar-Droz-Vincent model is the output of the many efforts to formulate the theory of the classical relativistic particle mechanics (see Refs. 4, 12, and 15 for reviews, and also Ref. 4. See Ref. 16 for reviews on the predictive mechanics, and see also Refs. 7 and 17), overcoming the difficulties introduced by the no-interaction theorem, ${ }^{18}$ which prevents the physical coordinates from being simultaneously covariant and canonical in the interacting case, in any of the forms of the dynamics introduced by Dirac. ${ }^{19}$ From here it emerges the dualism between the physical covariant coordinates $\left\{q^{\mu}\right\}$ and the phase-space canonical coordinates $\left\{x^{\mu}\right\}$. The models which use the firstclass constraints approach are expressed in terms of the coordinates $\left\{x^{\mu}\right\}$, in order to avoid the consequences of the nointeraction theorem, while the model formulated in the predictive approach are expressed in terms of the coordinates $\left\{q^{\mu}\right\}$. The work of Droz-Vincent, in particular Ref. 20 , establishes a bridge between the two approaches, and provides a connection between the above mentioned dualism and the many times formulation.

The present paper was originated by the wish to clarify these problems, avoiding the complications due to special relativity. We start from the classical nonrelativistic Newton's equations for $n$ particles, as a preliminary laboratory, deferring the quantum aspects as well as the physical interpretation, and the interpretation as a gauge theory to a future paper. The first step will be to get an $n$-time version of New- 
ton's equations of motion, which will be the nonrelativistic counterpart of the relativistic predictive equations, then we will put them in a first-order form.

In order to gain a canonical formulation, suitable for the quantization, we will give a generalization to $n$-times (nonautonomous case) of the classical Lie-König theorem ${ }^{21}$ for which the reader is also referred to Refs. 22 and 23 . In this way we will find all the $n$-time local (symplectic) structures, or better, the Poisson structures, which can be associated to the given equations of motion, and we will immediately find the dualism between the physical position $q^{i}\left(t^{i}\right)$ of the $i$ th particle and its canonical coordinate $x^{i}\left(t^{1}, t^{2}, \ldots, t^{n}\right)$. While the former only depends on its own time, the latter depends on all the $t^{i}$. Moreover we will get the $\boldsymbol{n}$-time generalization of the inverse problem in the calculus of variations, in the first-order formalism ${ }^{24}$ (see Ref. 25 for a review), and, as a by-product, we will get a nonrelativistic no-interaction theorem, and it will be possible to demonstrate the nonexistence of a predictive Lagrangian, independent on the accelerations, in the interacting case; only a singular Lagrangian can be defined.

The study of the invariance transformations of the $n$ time Newton equations in the first-order form will provide for an $n$-time generalization of the Currie-Hill conditions, ${ }^{26}$ for the Galilei algebra, as well as of the first Noether theorem, and for the conditions on the invariance transformations for being canonically implementable, with the chosen symplectic structure.

The final step will be to define an enlarged phase space $\bar{M}$, with the new canonical variables $t^{i}$ and $\epsilon_{i}$, times and energies, respectively, with $n$ first-class constraints. It will be shown that this is a coisotropic embedding ${ }^{27}$ in the phase space $\bar{M}$ of the original presymplectic manifold. ${ }^{28}$ (See, also Ref. 29 for a set of first-class constraints describing $n$ nonrelativistic free particles, and Refs. 12 and 30 for the case of two nonrelativistic interacting particles.) Finally, the DrozVincent method ${ }^{20}$ will allow the recovery of the physical position coordinates from the canonical ones.

It is our hope that, at the end of this paper, it will be clear that many features of the $n$-time approach are not peculiar of a relativistic theory, but, rather, they simply are more complicated in the relativistic case, with the result of hiding their basic simplicity. To reveal this simplicity we need a reformulation of Newton's equation of motion, which is probably useless for the applications, but it is inescapable for the present kind of problems.

As a matter of fact, the present analysis is quite general, and it could in principle be applied to any dynamical system. It is only necessary to specify the kinematical group of the theory, that is, for instance, the Poincaré group instead of the Galilei group. The only difference, which in practice becomes a real difficulty, is that in the present analysis the constraints are energy constraints, that is they are linear in the energies of the particles. This means that, in a relativistic theory, where the constraints are usually given in a covariant form, we should solve them in terms of the energies, and, in general, several local solutions will be possible. The present analysis must be separately applied to each of these solutions.
In Sec. II we will discuss the $n$-time approach to the equations of motion. We will explicitly develop a very simple model for two particles, for which we will give the explicit expression of the two-time forces in Appendix A.

In the same section we will discuss the canonical formulation of the dynamics.

In Sec. III the generalization to many independent variables of the classical Lie-Köning theorem will be outlined in order to establish, on general grounds, the existence of a canonical formulation. Some of the details are given in Appendix B. In Sec. IV we will discuss the invariances of the theory.

Finally, in Sec. V, the problem of the position coordinates and of their correlation with the canonical coordinates will be discussed.

Some of the present material was already presented in Refs. 30 and 31.

\section{THE $n$-TIME FORMULATIOIN OF THE DYNAMICS}

Given the equations of motion of a system of particles in a nonrelativistic theory

$$
m_{i} \ddot{\mathbf{q}}^{i}=\mathbf{F}^{i}\left(t, \mathbf{q}^{j}, \mathbf{v}^{j}\right), \quad i=1,2, \ldots, n,
$$

where $q^{i}$ are the positions of the particles and $t$ the time in a given inertial reference frame, it is always possible, in principle, to get an $n$-time formulation by eliminating the integration constants from their solutions and their first derivatives. Let us write the general solution of the system (2.1),

$$
\mathbf{q}^{i}=\mathbf{g}^{i}\left(t, c_{1}, c_{2}, \ldots, c_{6 n}\right),
$$

where $c_{1}, c_{2}, \ldots c_{6 n}$ are integration constants. In solution (2.2) we may choose a different time for each particle, that is

$$
\mathbf{q}^{i}\left(t^{i}\right)=\mathbf{g}^{i}\left(t^{i}, c_{1}, c_{2}, \ldots, c_{6 n}\right) .
$$

If we eliminate the integration constants $c_{1}, c_{2}, \ldots, c_{6 n}$ from Eqs. (2.3) and their derivatives

$$
\mathbf{v}^{i}\left(t^{i}\right)=\frac{d}{d t^{i}} \mathbf{q}^{i}\left(t^{i}\right)=\dot{\mathbf{g}}^{i}\left(t^{i}, c_{1}, c_{2}, \ldots, c_{6 n}\right),
$$

and substitute in

$$
\mathbf{a}^{i}\left(t^{i}\right)=\frac{d^{2}}{d t^{i 2}} \mathbf{q}^{i}\left(t^{i}\right)=\ddot{\mathbf{g}}^{i}\left(t^{i}, c_{1}, c_{2}, \ldots, c_{6 n}\right),
$$

we get the $n$-time equations of motion

$$
m_{i} \mathbf{a}^{i}\left(t^{i}\right)=\mathscr{F}^{i}\left(t^{j}, \mathbf{q}^{j}, \mathbf{v}^{j}\right) .
$$

Since the $i$ th lhs only depends on $t^{i}$, we must have

$$
\begin{aligned}
\frac{d}{d t^{j}} \mathscr{F}^{i} & =\left(\frac{\partial}{\partial t^{j}}+\mathbf{v}^{j} \cdot \frac{\partial}{\partial \mathbf{q}^{j}}+\mathbf{a}^{j} \cdot \frac{\partial}{\partial \mathbf{v}^{j}}\right) \mathscr{F}^{i} \\
& =\left(\frac{\partial}{\partial t^{j}}+\mathbf{v}^{j} \cdot \frac{\partial}{\partial \mathbf{q}^{j}}+\frac{\mathscr{F}^{j}}{m_{j}} \cdot \frac{\partial}{\partial \mathbf{v}^{j}}\right) \mathscr{F}^{i}=0,
\end{aligned}
$$

for $i \neq j$.

Equations (2.7) can be called predictivity conditions, and the $n$-times forces $\mathscr{F}^{i}$ predictive forces. This in order to agree with the literature on predictive mechanics quoted in the Introduction (see Refs. 7, 16, and 17). Putting $t^{1}=t^{2}=\cdots=t^{n}=t$ in Eq. (2.6), we must have

$$
\mathscr{F}^{i}\left(t, \mathbf{q}^{j}, \mathbf{v}^{j}\right)=\mathbf{F}^{i}\left(t, \mathbf{q}^{j}, \mathbf{v}^{j}\right) \text {. }
$$


Let us now consider the system (2.6): It seems difficult to get it from an action principle, or, more simply, to get the forces $\mathscr{F}^{i}$ from some potential, or even if possible, as is apparent from the example of the Appendix A, it will be very complicated. As a matter of fact, we will show in Sec. V that a second-order Lagrangian for the equations of motion (2.6) does not exist. So we will look for a possible canonical formulation in terms of other variables.

In Sec. III we will give a generalization to many variables $t^{i}(i=1,2, \ldots, n)$ of the classical Lie-König theorem, which asserts that, for any given set of first-order ordinary differential equations, it is always possible to find new variables, $\mathbf{x}^{i}$ and $\mathbf{p}^{i}$ (in place of the positions and velocities), and a function of them, $\mathbf{H}$, such that the set of equations is transformed to canonical form.

In our case the generalization of this theorem says that, given the set of first-order equations (now partial derivative equations)

$$
\begin{aligned}
& \frac{\partial \mathbf{q}^{i}}{\partial t^{j}}=\mathbf{v}^{i} \delta_{j}^{i}, \\
& m_{i} \frac{\partial \mathbf{v}^{i}}{\partial t^{j}}=\delta_{j}^{i} \mathscr{F}^{i}\left(t^{k}, \mathbf{q}^{k}, \mathbf{v}^{k}\right),
\end{aligned}
$$

it is always possible to find new variables $\mathbf{x}^{i}=\mathbf{x}^{i}\left(t^{k}, \mathbf{q}^{k}, \mathbf{v}^{k}\right)$ and $\mathbf{p}_{i}=\mathbf{p}_{i}\left(t^{k}, \mathbf{q}^{k}, \mathbf{v}^{k}\right)$, and besides $n$ functions $H_{i}=H_{i}\left(t^{k}, \mathrm{x}^{k}, \mathrm{p}_{k}\right)$ satisfying the integrability conditions

$$
\frac{\partial H_{i}}{\partial t^{j}}-\frac{\partial H_{j}}{\partial t^{i}}+\left\{H_{i}, H_{j}\right\}=0,
$$

where

$$
\{A, B\}=\sum_{i=1}^{n}\left(\frac{\partial A}{\partial \mathbf{x}^{i}} \cdot \frac{\partial B}{\partial \mathbf{p}_{i}}-\frac{\partial B}{\partial \mathbf{x}^{i}} \cdot \frac{\partial A}{\partial \mathbf{p}_{i}}\right),
$$

such that the system (2.9) can be written in canonical form:

$$
\begin{aligned}
& \frac{\partial \mathbf{x}^{i}}{\partial t^{j}}=\left\{\mathbf{x}^{i}, H_{j}\right\}, \\
& \frac{\partial \mathbf{p}_{i}}{\partial t^{j}}=\left\{\mathbf{p}_{i}, H_{j}\right\} .
\end{aligned}
$$

We leave to the next section the demonstration of this result. As a matter of fact, this theorem asserts the existence of at least one canonical formulation, but says very little for actual construction of the Hamiltonians $H_{i}$.

For the moment, we will assume the existence of $n$ functions $H_{i}$, satisfying the conditions (2.10), in order to formulate the action principle in canonical form.

Let us consider the following line integral:

$$
S=\int_{a(l)}^{b}\left(\mathbf{p}_{i} \cdot d \mathbf{x}^{i}-H_{i} d t^{i}\right),
$$

where $a$ and $b$ are two points in the $\left(t^{1}, t^{2}, \ldots, t^{n}\right)$ space and $l$ is a path connecting them.

$S$ is a functional of the $\mathbf{p}_{i}$ and $\mathbf{x}^{i}$ defined on the $\left(t^{1}, t^{2}, \ldots, t^{n}\right)$ space, for which the usual symplectic structure is assumed

$$
\left\{\mathbf{x}^{i m}, \mathbf{p}_{j}{ }^{n}\right\}=\delta^{m n} \delta_{j}^{i},
$$

where $m, n=1,2,3$ and $i, j=1,2, \ldots, n$, and the $H_{i}\left(t^{k}, \mathbf{x}^{k}, \mathbf{p}_{k}\right)$ satisfy the conditions $(2.10)$.
If we require $\delta S=0$ for an arbitrary variation of $\mathbf{p}_{i}=\mathbf{p}_{i}\left(t^{1}, t^{2}, \ldots, t^{n}\right)$ and $\mathbf{x}^{i}=\mathbf{x}^{i}\left(t^{1}, t^{2}, \ldots, t^{b}\right)$, which vanishes in $a$ and $b$, and for any choice of the path $l$, we get the following equations of motion:

$$
\begin{aligned}
d \mathbf{x}^{i} & =\left\{\mathbf{x}^{i}, H_{j}\right\} d t^{j}, \\
d \mathbf{p}_{i} & =\left\{\mathbf{p}_{i}, H_{j}\right\} d t^{j}
\end{aligned}
$$

(a sum over repeated indices is assumed), which are integrable in view of the conditions (2.10).

Equations (2.15) can be obtained on any given path $l$, in which case $d t^{j}=t^{\prime j}(\tau) d \tau$, where $\tau$ is any parameter for the path $l$, and the functions $t^{\prime j}(\tau)$ depend on the choice of $l$. Since Eqs. (2.15) are integrable, the canonical coordinates $\mathbf{x}^{i}$ and $\mathbf{p}_{i}$ exist as functions on the space $\left(t^{1}, t^{2}, \ldots, t^{n}\right)$ and not only on the path $l$. So they will coincide with the particular solution found on a given $l$, when the independent variables $t^{i}$ are restricted on $l$.

When $\mathbf{x}^{i}$ and $\mathbf{p}_{i}$ are solutions of the equations of motion (2.15), the canonical action $S$ does not depend on $l$. Indeed the one-form

$$
\theta=\mathbf{p}_{i} \cdot \mathbf{x}^{i}-H_{i} d t^{i},
$$

is closed, when Eqs. (2.15) hold

$$
d \theta=\frac{1}{2}\left(\frac{\partial H_{i}}{\partial t^{j}}-\frac{\partial H_{j}}{\partial t^{i}}+\left\{H_{i}, H_{j}\right\}\right) d t^{i} \wedge d t^{j}=0 .
$$

For any dynamical variable $\xi=\xi\left(t^{k}, \mathbf{x}^{k}, \mathbf{p}_{k}\right)$, the equation of motion reads

$$
d \xi=\left(\frac{\partial \xi}{\partial t^{i}}+\left\{\xi, H_{i}\right\}\right) d t^{i}
$$

Let us notice that in general we have that

$$
\mathbf{x}^{i}=\mathbf{x}^{i}\left(t^{1}, t^{2}, \ldots, t^{n}\right)
$$

and

$$
\mathbf{p}_{i}=\mathbf{p}_{i}\left(t^{1}, t^{2}, \ldots, t^{n}\right),
$$

whereas the positions $\mathbf{q}^{i}$ are only functions of the corresponding $t^{i}$. The only case in which the $\mathrm{x}^{i}$ only depend on $t^{i}$ happens when the $H_{i}$, for $i \neq j$, do not depend on the $\mathrm{p}_{i}$, for instance when the $i$ th particle is free.

This fact already shows that in general the canonical variables $\mathbf{x}^{i}$ cannot coincide with the positions $\mathbf{q}^{i}$. Only when the interaction between the particles vanishes, we can freely make the choice $\mathbf{x}^{i}=\mathbf{q}^{i}$.

The fact that the canonical variables $\mathbf{x}^{i}$ and the physical positions $\mathbf{q}^{i}$ do not coincide, apart from the free case, is common in the relativistic theories, where this fact is essentially a consequence of the no-interaction theorem. But we now see that it is not peculiar of relativity, but rather of a multitime approach.

We will discuss the relation between the positions $q^{i}$ and the canonical variables in Sec. V, where we will give the precise connection between the canonical formalism and the equations of motion (2.6).

As previously mentioned, the next section is devoted to the proof of the existence of a canonical formulation, once Eqs. (2.6) are given, that is of a generalization of the LieKönig theorem. 


\section{A GENERALIZATION OF THE LIE-KÖNIG THEOREM}

The Lie-König theorem ${ }^{21}$ was discussed and applied in connection with the relativistic dynamics of systems of particles by Hill $^{22}$ and Kerner. ${ }^{32}$

Here we will essentially follow the treatment given in Refs. 22 in order to look for a generalization of this theorem to many independent variables $\left\{t^{i}\right\}, i=1,2, \ldots, n$.

The equations of motion (2.6) can be written as a firstorder system as in Eqs. (2.9):

$$
\begin{aligned}
& \frac{\partial \mathbf{q}^{i}}{\partial t^{j}}=\mathbf{v}^{i} \delta_{j}^{i}, \\
& \frac{\partial \mathbf{v}^{i}}{\partial t^{j}}=\frac{1}{m_{i}} \delta_{j}^{i} \mathscr{F}^{i}\left(t^{k}, \mathbf{q}^{k}, \mathbf{v}^{k}\right),
\end{aligned}
$$

where $i, j=1,2, \ldots, n$.

The conditions (2.7) will now be written in the shorthand notation

$$
Y_{i} \mathscr{F}^{j}=0, \text { for } i \neq j,
$$

where

$$
Y_{i}=\frac{\partial}{\partial t^{i}}+\mathbf{v}^{i} \cdot \frac{\partial}{\partial \mathbf{q}^{i}}+\frac{\mathscr{F}^{i}}{m_{i}} \cdot \frac{\partial}{\partial \mathbf{v}^{i}} .
$$

Let us introduce a new notation,

$$
\begin{array}{ll}
\left\{y^{a}\right\}=\left\{\mathbf{q}^{h}\right\}, & \text { for } a=1,2, \ldots, N=3 n, \\
\left\{y^{a}\right\}=\left\{\mathbf{v}^{\prime}\right\}, & \text { for } a=N+1, N+2, \ldots, 2 N .
\end{array}
$$

With this notation the equations of motion (2.9) can be written

$$
\frac{\partial y^{a}}{\partial t^{i}}=h_{i}^{a}(y, t)
$$

where

$$
\begin{aligned}
& \left\{h_{i}^{a}\right\}=\left\{\mathbf{h}_{i}^{j}\right\}, \quad \mathbf{h}_{i}^{j}=\delta_{i}^{j} \mathbf{v}^{i}, \quad \text { for } a=1,2, \ldots, N, \\
& \left\{h_{i}^{a}\right\}=\left\{\mathbf{h}_{i}^{n+j}\right\}, \quad \mathbf{h}_{i}^{n+j}=\delta_{i}^{j}\left(1 / m_{i}\right) \mathscr{F}^{i}, \\
& \quad \text { for } a=N+1, \ldots, 2 N .
\end{aligned}
$$

The conditions ( 3.1 ) become

$$
Y_{i} h_{j}^{a}=0 \text {, for } i \neq j \text {, }
$$

where

$$
Y_{i}=\frac{\partial}{\partial t^{i}}+h_{i}^{a} \frac{\partial}{\partial y^{a}} .
$$

The conditions (3.6) are a particular case of the integrability conditions for the system (3.4), which is a Mayer system $^{33}$; the more general integrability conditions are

$$
Y_{i} h_{j}^{a}=Y_{j} h_{i}^{a} \text {. }
$$

From now on, if not otherwise specified, we will assume the more general conditions (3.8) in place of (3.6).

Let us now look for a variational principle giving the set of equations of motion (3.4) in the form

$$
\delta S=0,
$$

where

$$
S=\int_{a(l)}^{b} \theta, \quad \theta=U_{a} d y^{a}-V_{i} d t^{i},
$$

and where $l$ is a path in the space of the times $T=\left\{t^{i}\right\}$, $i=1,2, \ldots, n$, connecting $a=\left\{t_{(a)}^{i}\right\}$ to $b=\left\{t_{(b)}^{i}\right\}$.

The variational principle (3.9) will be required for all the variations of the functions

$$
y^{a}\left(t^{1}, t^{2}, \ldots, t^{n}\right)
$$

which vanish at the end points of the path $l, a$ and $b$, and for all the variations of the path $l$ with fixed endpoints.

At the end points $a$ and $b$ the functions $q^{l}\left(t^{1}, t^{2}, \ldots, t^{n}\right)$ will be considered as given, and the functions $v^{i}\left(t^{1}, t^{2}, \ldots, t^{n}\right)$ fixed, but undetermined. It is worth recalling that, when the more restricted integrability conditions (3.6) will hold, the variables $q^{i}$ will only depend on their own time $t^{i}$.

A variation of $y^{a}\left(t^{1}, t^{2}, \ldots, t^{n}\right)$ and of $l$ amounts in performing independent variations of $y^{a}$ and $t^{i}$.

If we introduce another notation

$$
\left\{y^{\alpha}\right\}=\left\{y^{a}, t^{i}\right\}, \quad \alpha=1,2, \ldots, 2 N+n,
$$

and put

$$
\begin{array}{ll}
U_{\alpha}=U_{a}, & \text { for } \alpha=1,2, \ldots, 2 N, \\
U_{\alpha}=-V_{i}, & \text { for } \alpha=2 N+i, i=1,2, \ldots, n,
\end{array}
$$

the one-form $\theta$ can be written

$$
\theta=U_{\alpha} d y^{\alpha} .
$$

The variation of this form gives rise to

$$
\delta \theta=\left(\frac{\partial U_{\alpha}}{\partial y^{\beta}}-\frac{\partial U_{\beta}}{\partial y^{\alpha}}\right) d y^{\alpha} \delta y^{\beta}+d\left(U_{\alpha} \delta y^{\alpha}\right),
$$

so the equations of motion corresponding to $\delta S=0$ will be

$$
\Gamma_{\alpha \beta} d y^{\alpha}=0,
$$

where we have defined

$$
\Gamma_{\alpha \beta}=\frac{\partial U_{\alpha}}{\partial y^{\beta}}-\frac{\partial U_{\beta}}{\partial y^{\alpha}} .
$$

Equations (3.14) must be identified with Eqs. (3.4). Since Eqs. (3.14), when explicitly written, are

$$
\begin{aligned}
& \Gamma_{a b} d y^{b}+\Gamma_{a i} d t^{i}=0 \\
& \Gamma_{i a} d y^{a}+\Gamma_{i j} d t^{j}=0
\end{aligned}
$$

this requires

$$
\left|\Gamma_{a b}\right| \neq 0 \quad(a, b=1,2, \ldots, 2 N),
$$

and

$$
\begin{aligned}
& \Gamma_{a b} h_{i}^{b}+\Gamma_{a i}=0, \\
& \Gamma_{i a} h_{j}^{a}+\Gamma_{i j}=0,
\end{aligned}
$$

or

$$
\begin{aligned}
& \Gamma_{a b} h_{i}^{b}=-\Gamma_{a i}, \\
& \Gamma_{a b} h_{i}^{a} h_{j}^{b}=\Gamma_{i j} .
\end{aligned}
$$

The first of these equations gives the "forces" $h_{i}^{a}$ in terms of $\Gamma$,

$$
h_{i}^{a}=-\left(\Gamma^{-1}\right)^{a b} \Gamma_{b i} \text {. }
$$

The condition (3.17) implies that the two-form

$$
\omega=d \theta=-\frac{1}{2} \Gamma_{\alpha \beta} d y^{\alpha} \wedge d y^{\beta}
$$

has rank $2 N$, because $\omega$ can also be written as

$$
\omega=-\frac{1}{2} \Gamma_{a b} \theta^{a} \wedge \theta^{b},
$$


where

$$
\theta^{a}=d y^{a}-h_{i}^{a} d t^{i} .
$$

The system $\theta^{a}=0$, which is the set of equations of motion (3.4), is completely integrable due to the integrability conditions (3.8). This can also be verified with the Frobenius theorem, since

$$
d \theta^{a}=\left(\frac{\partial h_{i}^{a}}{\partial y^{b}} d t^{i}\right) \wedge \theta^{b},
$$

where Eq. (3.8) was used.

Since

$$
i_{Y_{i}} \theta^{a}=0 \text {, }
$$

where $i_{Y_{I}}$ is the contraction with respect the vector field $Y_{i}$ (see for instance Ref. 34) the dual form of the Eq. (3.23) is in terms of the characteristic vector fields $Y_{i}$, that is

$$
\left[Y_{i}, Y_{j}\right]=0 \text {. }
$$

The fields $Y_{i}$ span the kernel distribution of $\omega$ since

$$
i_{Y_{i}} \omega=0 \text {, }
$$

so the kernel distribution of $\omega$ is $n$-dimensional, the $Y_{i}$ being linearly independent.

From Eq. (3.21), we have that, on the motion,

$$
\omega=d \theta=0 \text {. }
$$

This has the important consequence that the action $S$, when evaluated on the motion, does not depend on the path $l$, but only on the end points $a$ and $b$. Indeed, using Stokes' theorem, we have that for two paths $l$ and $l^{\prime}$ with the same end points

$$
\left(\int_{a\left(I^{\prime}\right)}^{b}-\int_{a(l)}^{b}\right) \theta=\int_{\partial D} \theta=\int_{D} d \theta=0,
$$

where $D$ is the domain, in the space of times $T=\{t\}$, bounded by $l$ ' and $l$.

In the notation ( 3.11 ), by setting

$$
\begin{aligned}
& h_{i}^{\alpha}=h_{i}^{a}, \quad \text { for } \alpha=a, \\
& h_{i}^{\alpha}=\delta_{i}^{j}, \quad \text { for } \alpha=2 N+j,
\end{aligned}
$$

we may write

$$
Y_{i}=h_{i}^{\alpha} \frac{\partial}{\partial y^{\alpha}}
$$

and the integrability conditions (3.8) can now be written

$$
Y_{i} h_{j}^{\alpha}=Y_{j} h_{i}^{\alpha} \text {, }
$$

since, for $\alpha=2 N+1, \ldots, 2 N+n$, they become identities. In the case of the restricted integrability conditions (3.1), the right-hand side of Eq. (3.30) is zero. Equations (3.4) can be written

$$
d y^{\alpha}=h_{i}^{\alpha} d t^{i},
$$

and the equations for $\Gamma_{\alpha \beta}$ can be summarized as follows:

$$
\begin{aligned}
& \Gamma_{\alpha \beta}=-\Gamma_{\beta \alpha}, \\
& \frac{\partial \Gamma_{\alpha \beta}}{\partial y^{\gamma}}+\text { cyclic }=0, \\
& \Gamma_{\alpha \beta} h_{i}^{\beta}=0,
\end{aligned}
$$

where the first and the second equations are a consequence of Eq. (3.15), and the last collects Eqs. (3.18).

Together with these equations we must remember the requirement

$$
\left|\Gamma_{a b}\right| \neq 0, \text { for } a, b=1,2, \ldots, 2 N \text {. }
$$

Equations (3.32) and (3.33) are merely identities: this follows from the definition of $\Gamma_{\alpha \beta}$ in Eq. (3.16). Nevertheless they can be assumed as defining equations for $\Gamma_{\alpha \beta} . \mathrm{Ob}-$ serve that from these equations and Eq. (3.34), and using the integrability conditions (3.8), the components $\Gamma_{a i}$ and $\Gamma_{i j}$ can be eliminated, obtaining two equations for $\Gamma_{a b}$ solely

$$
\begin{aligned}
& Y_{i} \Gamma_{b c}=\left(\Gamma_{a b} \partial_{c}-\Gamma_{a c} \partial_{b}\right) h_{i}^{a}, \\
& \frac{\partial \Gamma_{a b}}{\partial y^{c}}+\text { cyclic }=0 .
\end{aligned}
$$

Equations (3.33), when written explicitly for the components $\Gamma_{a b}, \Gamma_{a i}$, and $\Gamma_{i j}$, reveal the self-adjoint nature of Eqs. (3.14). Indeed these equations split in four sets of equations, of which two are Eqs. (3.37) and

$$
\frac{\partial \Gamma_{a b}}{\partial t^{i}}+\frac{\partial \Gamma_{i a}}{\partial y^{b}}+\frac{\partial \Gamma_{b i}}{\partial y^{a}}=0,
$$

while the two other sets are identically satisfied, due to Eqs. (3.36) and (3.8).

The reader is referred to Ref. 35 for the definition of selfadjoint systems in the one-time case.

Let us notice that, when the restricted form (3.6) of the integrability conditions holds, to each solution $\Gamma_{a b}$ of Eqs. (3.36) and (3.37), such that its restriction at equal times has the form

$$
\left\|\left.\Gamma_{a b}\right|_{t^{1}, t^{2}, \ldots, t^{n}=t}\right\|=\left(\begin{array}{cc}
\beta & -\alpha \\
\alpha & 0
\end{array}\right),
$$

where $\alpha$ and $\beta$ are $N \times N$ matrices, and $\alpha=\alpha^{T}, \beta=-\beta^{T}$, and where the zero sector refers to

$$
\frac{\partial U_{i+n}^{m}}{\partial v^{k j}}-\frac{\partial U_{j+n}^{k}}{\partial v^{m i}} \quad(m, k=1,2,3),
$$

a solution of the inverse problem of the calculus of variations is associated, that is there exists a Lagrangian for Eqs. (2.1), and $\alpha$ and $\beta$ can be expressed as

$$
\begin{aligned}
& \alpha_{i m, j k}=\frac{\partial^{2} L}{\partial \dot{q}^{i m} \partial \dot{q}^{j k}}, \\
& \beta_{i m j k}=\frac{\partial^{2} L}{\partial q^{i m} \partial \dot{q}^{j k}}-\frac{\partial^{2} L}{\partial q^{i k} \partial \dot{q}^{i m}},
\end{aligned}
$$

where $\dot{q}^{i m}=v^{i m}$.

As shown in Ref. 24 this condition is equivalent to the Helmholtz conditions in the Douglas form, as are expressed in the first-order formalism (see Ref. 36). The same results are also given in Ref. 37, and, in a more geometric way, in Ref. 38 by using a variant of the one-forms (3.22). As there are inequivalent classes of solutions for $\Gamma_{a b}$ (see in the following of this section), the system (2.1) will have no one, one or several inequivalent (or $s$-equivalent) Lagrangians, according to how many solutions $\Gamma_{a b}$ admit the form (3.39) (see Ref. 25).

Coming back to the general discussion, with the integrability conditions in the general form (3.30), and before in- 
troducing local canonical variables, let us discuss the structure of the system (3.18) or (3.34), for $U_{a}$ and $V_{i}$, for given forces $h_{i}^{a}(y, t)$.

First of all let us notice that Eq. (3.34) is invariant under the transformation

$$
U_{\alpha} \rightarrow U_{\alpha}+\frac{\partial \Phi}{\partial y^{\alpha}},
$$

where $\Phi$ is any function of the $\left\{y^{\alpha}\right\} \equiv\left\{y^{a}, t^{i}\right\}$.

Under this transformation $\theta$ transforms as

$$
\theta \rightarrow \theta+d \Phi \text {, }
$$

which does not modify the equations of motion. This transformation will be called a canonical transformation.

To be more general, we have to observe that even the transformation

$$
\theta \rightarrow \theta+g_{i}\left(t^{1}, t^{2}, \ldots, t^{n}\right) d t^{i},
$$

does not modify the equations of motion, corresponding to the first of Eqs. (3.16), since there the $U_{i}=-V_{i}$ appear differentiated with respect to the $y^{a}$ only, but it breaks the independence on the path $l$ of the action, expressed by the second of Eqs. (3.16) since it is this equation that appears in $\delta \theta$ as a coefficient of $\delta t^{i}$. So we will not consider any more such a transformation as a true invariance of the theory.

Coming back to the transformation (3.40), we observe that a solution of Eqs. (3.34), recalling the definition (3.15), can be written

$$
\begin{aligned}
& U_{a}=U_{a}^{(0)}+\frac{\partial \Phi}{\partial y^{a}}, \\
& V_{i}=-U_{2 N+i}=U_{a}^{(0)} h_{i}^{a}-\frac{\partial \Phi}{\partial t^{i}},
\end{aligned}
$$

with $U_{a}^{(0)}$ a solution of the homogeneous equation

$$
Y_{i} U_{a}^{(0)}+U_{b}^{(0)} \frac{\partial h_{i}^{b}}{\partial y^{a}}=0
$$

satisfying the condition

$$
\left|\frac{\partial U_{a}^{(0)}}{\partial y^{b}}\right| \neq 0 \text {. }
$$

This is a consequence of the following facts, which are demonstrated in Appendix B: if we put

$$
V_{i}=-U_{2 N+i}=U_{a} h_{i}^{a}+\lambda_{i},
$$

it follows for $\lambda_{i}$,

$$
Y_{i} \lambda_{j}=Y_{j} \lambda_{i} .
$$

This in turn implies that $\lambda_{i}$ must be of the form

$$
\lambda_{i}=-Y_{i} \Phi,
$$

with $\Phi$ any arbitrary function. Finally, it is shown in Appen$\operatorname{dix} B$ that

$$
U_{a}-\frac{\partial \Phi}{\partial y^{a}}
$$

satisfies Eq. (3.44), from which we have

$$
U_{a}=U_{a}^{(0)}+\frac{\partial \Phi}{\partial y^{a}},
$$

and thus

$$
V_{i}=U_{a}^{(0)} h_{i}^{a}-\frac{\partial \Phi}{\partial t^{i}} .
$$

Equation (3.44) is discussed in Appendix B.

With the solution (3.43), the one-form $\theta$ can be written

as

$$
\theta=\theta^{(0)}+d \Phi,
$$

where

$$
\theta^{(0)}=U_{a}^{(0)} d y^{a}-V_{i}^{(0)} d t^{i}=U_{a}^{(0)} \theta^{a},
$$

because

$$
V_{i}^{(0)}=U_{a}^{(0)} h_{i}^{a} .
$$

The solution (3.43) shows that any independent solution $U_{a}^{(0)}$ of Eq. (3.44) determines a set of solutions connected by canonical transformations (3.40).

Clearly Eqs. (3.43) define an equivalence relation, and inequivalent choices of $\Gamma$ (with $\left\|\Gamma_{a b}\right\|$ not singular) will correspond to disjoint sets of solutions for $U_{\alpha}$, each element of one of these disjoint sets being connected to another of the same set by a canonical transformation.

The situation described here is completely analogous to that of the one-time case, analyzed in Ref. 22.

Now we can demonstrate that each disjoint set has a different symplectic structure, giving to our equations of motion (3.4), or (2.9), the form of canonical equations of motion, which is the main task of the present section. The crucial result is that the two-form $\omega=d \theta$ has rank $2 N$, as stressed after Eq. (3.20). On the other hand $\omega$ is closed, so from the (generalized) Darboux theorem, ${ }^{34}$ we know that local coordinates exist such that

$$
\omega=d \overline{\mathbf{p}}_{i} \wedge d \overline{\mathbf{x}}^{i} \quad(i=1,2, \ldots, n),
$$

from this we get

$$
\begin{gathered}
d\left(\theta-\overline{\mathbf{p}}_{i} d \overline{\mathbf{x}}^{i}\right)=0, \\
\text { or } \quad \\
\theta=\overline{\mathbf{p}}_{i} d \overline{\mathbf{x}}^{i}+d \phi,
\end{gathered}
$$

where $\phi$ is a function of $\overline{\mathbf{x}}^{i}, \overline{\mathbf{p}}_{i}$, and $t^{i}$.

Equation (3.53) shows that, for each inequivalent choice of $\Gamma_{\alpha \beta}$, we have a (different) symplectic structure. The coordinates $\overline{\mathbf{p}}_{i}$ and $\overline{\mathbf{x}}^{i}$ are a choice of the Jacobi coordinates (initial data), for which the Hamiltonians are vanishing. Let us now go back to a general choice of canonical variables $\mathbf{x}^{i}$ and $\mathbf{p}_{i}$, by performing a (backward) Jacobi transformation.

If we take $\phi$ to be any function of $\overline{\mathbf{x}}^{i}$ and $t^{i}$, and of a set of $N$ new variables $\mathbf{x}^{i}, \phi=\phi\left(\mathbf{x}^{i}, \overline{\mathbf{x}}^{i}, t^{i}\right)$, with the only requirement that

$$
|| \frac{\partial^{2} \phi}{\partial \mathbf{x}^{i} \partial \overline{\mathbf{x}}^{j}}|| \neq 0
$$

we may put

$$
\frac{\partial \phi}{\partial \overline{\mathbf{x}}^{i}}=-\overline{\mathbf{p}}_{i},
$$

and 
$\frac{\partial \phi}{\partial t^{i}}=-\widetilde{H}_{i}(\mathbf{x}, \overline{\mathbf{x}}, t)$,

$\frac{\partial \phi}{\partial \mathbf{x}^{i}}=\mathbf{p}_{i}$,

Condition (3.54) allows to invert the last equation (3.56), to get $\overline{\mathbf{x}}^{i}$ as a function of $\mathbf{x}^{i}, \mathbf{p}_{i}$, and $t_{i}$

$\overline{\mathbf{x}}^{i}=\mathbf{g}^{i}(\mathbf{x}, \mathbf{p}, t)$.

Inserting this expression in $\widetilde{H}_{i}$ we get $n$ new functions of $\mathbf{x}^{i}, \mathbf{p}_{i}$, and $t^{i}$

$$
H_{i}(\mathbf{x}, \mathbf{p}, t)=\widetilde{H}_{i}(\mathbf{x}, \mathbf{g}(\mathbf{x}, \mathbf{p}, t), t),
$$

which will be our Hamiltonians. The first equation (3.56) can now be written

$$
\frac{\partial \phi}{\partial t^{i}}+H_{i}\left(\mathbf{x}, \frac{\partial \phi}{\partial \mathbf{x}}, t\right)=0
$$

It is easily verified that the Hamiltonians so determined satisfy the following integrability conditions:

$$
\frac{\partial H_{i}}{\partial t^{j}}-\frac{\partial H_{j}}{\partial t^{i}}+\left\{H_{i}, H_{j}\right\}=0,
$$

where the Poisson bracket is defined with respect to the new canonical coordinates $\mathbf{x}$ and $\mathbf{p}$ :

$$
\{A, B\}=\sum_{i=1}^{n}\left(\frac{\partial A}{\partial \mathbf{x}^{i}} \cdot \frac{\partial B}{\partial \mathbf{p}_{i}}-\frac{\partial B}{\partial \mathbf{x}^{i}} \cdot \frac{\partial A}{\partial \mathbf{p}_{i}}\right) .
$$

Indeed, from Eqs. (3.59) we get

$$
\begin{aligned}
\frac{\partial^{2} \dot{\phi}}{\partial t^{j} \partial t^{i}} & =-\frac{\widetilde{H}_{i}(\mathbf{x}, \overline{\mathbf{x}}, t)}{\partial t^{j}} \\
& =-\frac{\partial H_{i}}{\partial t^{j}}-\frac{\partial H_{i}}{\partial \mathbf{p}_{k}} \cdot \frac{\partial}{\partial t^{j}} \frac{\partial \phi}{\partial \mathbf{x}^{k}} \\
& =-\frac{\partial H_{i}}{\partial t^{j}}+\frac{\partial H_{i}}{\partial \mathbf{p}_{k}} \cdot\left(\frac{\partial H_{j}}{\partial \mathbf{x}^{k}}+\frac{\partial H_{j}}{\partial \mathbf{p}_{l}} \cdot \frac{\partial^{2} \phi}{\partial \mathbf{x}^{k} \partial \mathbf{x}^{j}}\right),
\end{aligned}
$$

from which

$$
\frac{\partial^{2} \phi}{\partial t^{j} \partial t^{i}}-\frac{\partial^{2} \phi}{\partial t^{i} \partial t^{j}}=-\frac{\partial H_{i}}{\partial t^{j}}+\frac{\partial H_{j}}{\partial t^{i}}-\left\{H_{i}, H_{j}\right\}=0 .
$$

Let us stress that in this way we may generate sets of $n$ functions $H_{i}$ satisfying Eqs. (3.60), by simply choosing a function $\phi(\mathbf{x}, \overline{\mathbf{x}}, t)$, satisfying the condition (3.54).

The one-form $\theta$ becomes

$$
\begin{aligned}
\theta & =\overline{\mathbf{p}}_{i} d \overline{\mathbf{x}}^{i}+d \phi \\
& =\overline{\mathbf{p}}_{i} d \overline{\mathbf{x}}^{i}-\overline{\mathbf{p}}_{i} d \overline{\mathbf{x}}^{i}+\mathbf{p}_{i} d \mathbf{x}^{i}-H_{i} d t^{i},
\end{aligned}
$$

that is

$$
\theta=\mathbf{p}_{i} d \mathbf{x}^{i}-H_{i}(\mathbf{x}, \mathbf{p}, t) d t^{i},
$$

which is the local expression for $\theta$ we were looking for. This is the generalization to $n$ times of the Poincaré-Cartan oneform. The one-time evolution vector field $Y$, which is the solution of the equations

$$
i_{Y} \omega=0, \quad i_{Y} d t=1 \quad(\omega=d \theta),
$$

is now replaced by the $n$ vector fields $Y_{i}$, satisfying Eqs. (3.26), and

$$
i_{Y_{i}} d t^{j}=\delta_{i}^{j} .
$$

Clearly the inverse procedure, that is, given the functions $H_{i}$, satisfying the integrability conditions $(3.60)$, to determine the function $\phi(\mathbf{x}, \mathbf{p}, t)$, amounts in determining a complete integral of the $\boldsymbol{n}$ (integrable) Jacobi equations (3.59).

In Appendix B the relation between the coordinates $\overline{\mathbf{x}}$ and $\bar{p}$ and the integration constants of the original equations of motion (3.4) is discussed.

If we compare the expression of $d \theta$ as given by Eq. (3.62) with that given by Eq. (3.10), we get the following equality:

$$
\Gamma_{a b}=\frac{\partial U_{a}}{\partial y^{b}}-\frac{\partial U_{b}}{\partial y^{a}}=\left[y^{a}, y^{b}\right],
$$

where [ , ] is the Lagrange bracket

$$
\left[y^{a}, y^{b}\right]=\frac{\partial \mathbf{x}^{i}}{\partial y^{a}} \cdot \frac{\partial \mathbf{p}_{i}}{\partial y^{b}}-\frac{\partial \mathbf{x}^{i}}{\partial y^{b}} \cdot \frac{\partial \mathbf{p}_{i}}{\partial y^{a}},
$$

where $\mathbf{x}^{i}$ and $\mathbf{p}_{i}$ are considered functions of the variables $y^{a}$ and $t^{i}$.

The first equation (3.63) implies

$$
\left\{y^{a}, y^{b}\right\}=-\left(\Gamma^{-1}\right)^{a b},
$$

where we remember the condition (3.17), that is the hypothesis that $\left\|\Gamma_{a b}\right\|$ be nonsingular. Equation (3.65) follows from

$$
\left[y^{a}, y^{b}\right]\left\{y^{c}, y^{b}\right\}=\delta^{a c} .
$$

The first of Eqs. (3.18) gives

$$
h_{i}^{a}=-\left(\Gamma^{-1}\right)^{a b} \Gamma_{b i},
$$

where we have taken into account that

$$
\left\{y^{a}, y^{b}\right\} \frac{\partial F}{\partial y^{b}}=\left\{y^{a}, F\right\} .
$$

If we substitute the expression (3.67) into the expression of $\Gamma_{a i}$ of Eq. (3.63), we get the forces in canonical form

$$
h_{i}^{a}=\frac{\partial y^{a}}{\partial t^{i}}+\left\{y^{a}, H_{i}\right\} \text {, }
$$

which can also be written

$$
h_{i}^{\alpha}=Y_{i} y^{\alpha}=\left(\frac{\partial}{\partial t^{i}}+\left\{\cdot, H_{i}\right\}\right) y^{\alpha},
$$

from which we get the action of $Y_{i}$ on any function of $\left\{\mathbf{x}^{i}, \mathbf{p}_{i}, t^{i}\right\}$ :

$$
Y_{i}=h_{i}^{\alpha} \frac{\partial}{\partial y^{\alpha}}=\frac{\partial}{\partial t^{i}}+\left\{\cdot, H_{i}\right\} .
$$

This equation gives the canonical form of the vector fields, which span the kernel distribution of the two-form $\omega$.

Equation (3.60) is recovered from $\Gamma_{i j}$, using Eq. (3.18').

We see that $\Gamma_{a b}$ determines the symplectic structure of the theory, as already observed, and that $\Gamma_{a b}$ is a canonical invariant, which determines the canonical sector on which the original $n$-time theory is represented.

The equations of motion can now be obtained as the equations of the integral curves of the vector fields $Y_{i}$. Indeed, by solving (see, for instance, Ref. 34)

$$
i_{X} \omega=0 \text {, }
$$


we find for $\boldsymbol{X}$

$$
X=c^{i} Y_{i}=c^{i}\left(\frac{\partial}{\partial t^{i}}+\left\{\cdot, H_{i}\right\}\right),
$$

for any choice of the functions $c^{i}$.

The integral curves of the vector fields $Y_{i}$ have the equations

$$
\begin{aligned}
& d \mathbf{x}^{i}=\left\{\mathbf{x}^{i}, H_{j}\right\} d t^{j}, \\
& d \mathbf{p}_{i}=\left\{\mathbf{p}_{i}, H_{j}\right\} d t^{j},
\end{aligned}
$$

which are the $n$-time canonical equations of motion. For any canonical observable $F(\mathbf{x}, \mathbf{p}, t)$ we get

$$
d F=\left(\frac{\partial F}{\partial t^{i}}+\left\{F, H_{i}\right\}\right) d t^{i} .
$$

Equations (3.60) now become the integrability conditioins for Eqs. (3.73).

Observe that the coordinates $\mathbf{x}^{i}$ and $\mathbf{p}_{i}$ are now, on the motion, functions of all the times $t^{1}, t^{2}, \ldots, t^{n}$, contrary to the original positions $\mathbf{q}^{i}$, which are functions of their own time $t^{i}$ only.

If we restrict the $\mathbf{x}^{i}$ and the $\mathbf{p}_{i}$ of the given symplectic structure $\{$,$\} associated to a U_{\alpha}$, to $t^{1}=t^{2}=\cdots=t^{n}=t$, Eq. (3.73) give the usual one-time Hamilton's equations of motion, with

$$
H=\sum_{i=1}^{n} H_{i}
$$

If we consider the case of the restricted integrability conditions (3.6), when, to the given canonical sector $U_{\alpha}^{(0)}$, a $\Gamma_{a b}$ is associated, which at equal times satisfies Eq. (3.39), that is

$$
\left\|\left.\Gamma_{a b}\right|_{t^{1}, t^{2}, \ldots, t^{n}=t}\right\|=\left(\begin{array}{cc}
\beta & -\alpha \\
\alpha & 0
\end{array}\right),
$$

we have a Lagrangian for Eq. (2.1), and its Hessian is given by the matrix $\alpha$, so that $\operatorname{det} \alpha \neq 0$. Then

$$
\left(\Gamma^{-1}\right)^{a b}=\left(\begin{array}{cc}
0 & \alpha^{-1} \\
-\alpha^{-1} & \alpha^{-1} \beta \alpha^{-1}
\end{array}\right),
$$

which implies

$$
\left\{q^{m i}, q^{k}\right\}=0, \quad \text { at } t^{1}=t^{2}=\cdots=t^{n}=t .
$$

We can then make the identification

$$
\mathbf{q}^{i}=\mathbf{x}^{i}, \quad \text { at } t^{1}=t^{2}=\cdots=t^{n}=t,
$$

and, via the Legendre transformation, we may recover the standard one-time Hamiltonian formalism, with

$$
\begin{aligned}
& H=\sum_{i=1}^{n} H_{i} . \\
& \text { As } \mathbf{q}^{i}=\mathbf{q}^{i}\left(t^{i}\right), \text { it follows that } \\
& \left\{q^{m i}\left(t^{i}\right), q^{k i}\left(t^{i}\right)\right\}
\end{aligned}
$$

does not depend upon the time $t^{j}$, for $j \neq i$ : therefore its vanishing at equal times implies

$$
\left\{q^{m i}\left(t^{i}\right), q^{k i}\left(t^{i}\right)\right\}=0,
$$

also in the $n$-time case.

Vice versa

$$
\left\{q^{m i}, q^{k j}\right\}=0 \quad \text { at } t^{1}=t^{2}=\cdots=t^{n}=t,
$$

implies, considering Eq. (3.64),

$$
\begin{aligned}
\left(\Gamma^{-1}\right)^{a b} & =\left(\begin{array}{ll}
0 & a \\
b & c
\end{array}\right) \Rightarrow\left\|\left.\Gamma_{a b}\right|_{t^{\prime}=t^{2}=\cdots t^{n}=t}\right\| \\
& =\left(\begin{array}{cc}
-b^{-1} c a^{-1} & b^{-1} \\
a^{-1} & 0
\end{array}\right),
\end{aligned}
$$

for those symplectic structures for which det $a \neq 0$, det $b \neq 0$; but, from

$$
\Gamma_{a b}=-\Gamma_{b a}
$$

we get

$$
a^{-1}=-b^{-1}
$$

so we recover Eq. (3.39) with

$$
\alpha=b^{-1} \text { and } \beta=b^{-1} c b^{-1} \text {. }
$$

Instead, for a canonical sector $U_{\alpha}^{(0)}$, whose $\Gamma_{a b}$ does not satisfy Eq. (3.39), there is no Lagrangian for Eq. (2.1), giving rise to this symplectic structure, restricted to equal times, via the Legendre transformation. In general, we now have

$$
\left\{q^{m i}, q^{k j}\right\} \neq 0
$$

even at equal times, and it is always $q^{i} \neq x^{i}$. The only way to define a Hamiltonian is by using Eq. (3.75).

This is a constructive way to get the symplectic structures and the Hamiltonians for the original system of equations of motion, which do not admit a Lagrangian.

Let us conclude this section by showing that the $n$-times formalism becomes the usual one in the free case, and for equal times, with a suitable choice of the solution of the equations for $U_{a}$ and $V_{i}$.

In the free case we have

$$
\left\{h_{i}^{a}\right\} \equiv\left\{\mathbf{h}_{i}^{j}\right\}, \quad \mathbf{h}_{i}^{j}=\delta_{i}^{j} \mathbf{v}^{i} \text { for } a=1,2, \ldots, N,
$$

and

$$
\left\{h_{i}^{a}\right\}=0, \text { for } a=N+1, N+2, \ldots, 2 N \text {. }
$$

It is easily seen that a particular solution of Eqs. (3.17) and (3.18) for $U_{a}$ and $V_{i}$ is

$$
\begin{aligned}
& \left\{U_{a}\right\}=\left\{m_{i} \mathbf{v}^{i}\right\}, \quad \text { for } a=1,2, \ldots, N, \\
& \left\{U_{a}\right\}=0, \text { for } a=N+1, N+2, \ldots, 2 N,
\end{aligned}
$$

and

$$
V_{i}=\frac{1}{2} m_{i}\left(\mathbf{v}^{i}\right)^{2} \text {. }
$$

The one-form $\theta$ becomes

$$
\theta=\sum_{i=1}^{n}\left(m_{i} \mathbf{v}^{i} d \mathbf{q}^{i}-\frac{1}{2} m_{i}\left(\mathbf{v}^{i}\right)^{2} d t^{i}\right)
$$

The choice (3.78) can also be used in the interacting case, when equal times are chosen $t^{1}=t^{2}=\cdots=t^{n}=t$, if the interaction Hamiltonians only depend on the positions, and not on the velocities. In this case we must linearly combine the equations of motion with equal coefficients, in order to restrict to the chosen path $t^{i}=t$, in the space of the times. We get

$$
V=\sum_{i=1}^{n} V_{i}=\frac{1}{2} \sum_{i=1}^{n} m_{i}\left(\mathbf{v}^{i}\right)^{2}+W\left(\mathbf{q}^{i}, t\right)
$$

The one-form $\theta$ becomes 


$$
\theta=\sum_{i=1}^{n} m_{i} \mathbf{v}^{i} d \mathbf{q}^{i}-V d t,
$$

which is the usual one.

\section{INVARIANCE TRANSFORMATIONS}

In this section the infinitesimal transformations, which leave the equations of motion invariant, are considered. The invariance conditions for any infinitesimal transformation of the coordinates and the conditions for the existence of the corresponding canonical generators are established. These canonical generators are constant of the motion (Noether theorem), and they close a Poisson algebra, providing a canonical realization of the Lie algebra of the group of transformation under consideration, under the condition of a vanishing two-cohomology group.

All this is well known in the one-time case (see for instance Refs. 34 and 39).

The equations of motion (3.31)

$$
d y^{\alpha}=h_{i}^{\alpha} d t^{i},
$$

where $\alpha=1,2, \ldots, 2 N+n$ and $N=3 n$, as already observed, are the characteristic system for the set of equations in the unknown $f(y)$

$$
Y_{i} f(y)=0 \text {. }
$$

An infinitesimal transformation

$$
y^{\alpha} \rightarrow y^{\alpha}=y^{\alpha}+\epsilon g^{\alpha}(y)
$$

will be an invariance transformation of the equations of motion $(4.1)$ if

$$
\left[L_{g}, Y_{i}\right]=\lambda_{i j} Y_{j},
$$

where $L_{g}$ is the vector field which performs the transformation (4.3) on the arguments of any function:

$$
L_{g}=g^{\alpha} \frac{\partial}{\partial y^{\alpha}} \text {. }
$$

Now, the lhs of Eq. (4.4) is

$$
\begin{aligned}
{\left[L_{g}, Y_{i}\right] } & =\left(L_{g} h_{i}^{\alpha}-Y_{i} g^{\alpha}\right) \frac{\partial}{\partial y^{\alpha}} \\
& =\left(L_{g} h_{i}^{a}-Y_{i} g^{a}+\left(Y_{i} g^{j}\right) h_{j}^{a}\right) \frac{\partial}{\partial y^{a}}-\left(Y_{i} g^{j}\right) Y_{j},
\end{aligned}
$$

and we get

$$
\begin{aligned}
& {\left[L_{g}, Y_{i}\right]+\left(Y_{i} g^{j}\right) Y_{j}} \\
& \quad=\left(L_{g} h_{i}^{a}-Y_{i} g^{\alpha}+\left(Y_{i} g^{j}\right) h_{j}^{a}\right) \frac{\partial}{\partial y^{\alpha}} \\
& \quad=\left(L_{g} h_{i}^{\alpha}-Y_{i} g^{\alpha}+\left(Y_{i} g^{j}\right) h_{j}^{\alpha}\right) \frac{\partial}{\partial y^{\alpha}} .
\end{aligned}
$$

Therefore, Eqs. (4.4) become

$$
\left[L_{g}, Y_{i}\right]=-\left(Y_{i} g^{j}\right) Y_{j},
$$

and it implies

$$
L_{g} h_{i}^{\alpha}-Y_{i} g^{\alpha}+h_{j}^{\alpha} Y_{i} g^{j}=0 .
$$

These conditions could be called the $n$-time Currie-Hill conditions, as they are the generalization of the Currie-Hill conditions for the invariance under the Lorentz group in the one-time case. ${ }^{26}$
The same conditions (4.7) can be obtained by requiring that the Lie derivative, with respect to $L_{8}$, of the one-forms $\theta^{a}$, defined in Eqs. (3.22), be proportional to the $\theta^{a}$ themselves.

If the transformations (4.3) form a Lie group $G$, the corresponding vector fields $L_{g}$ will close a Lie algebra $\mathscr{G}$. If $L_{1}, L_{2}, \ldots$, is a basis of $\mathscr{G}$, we will have

$$
\left[L_{m}, L_{k}\right]=c_{m k}^{l} L_{l} \text {, }
$$

where $c_{m k}^{l}$ are the structure constants of $\mathscr{G}$.

An infinitesimal transformation is a canonical transformation if it transforms the one-form $\theta$ of Eq. (3.13) as

$$
\theta \rightarrow \theta^{\prime}=\theta+\epsilon d \Omega .
$$

Therefore, the transformations generated by the vector fields $L_{g}$ will be canonical if

$$
\mathscr{L}_{L_{\mathrm{x}}} \theta=d \Omega_{\mathrm{g}},
$$

where $\mathscr{L}_{L_{g}}$ is the Lie derivative with respect to the vector fields $L_{g}\left(\mathscr{L}_{L_{g}}=i_{L_{g}} d+d i_{L_{g}}\right)$. From Eq. (4.10) we get

$$
d\left(\mathscr{L}_{L_{\mathrm{g}}} \theta\right)=0 \text {, }
$$

or

$$
\mathscr{L}_{L_{g}} \omega=d\left(i_{L_{g}} \omega\right)=0,
$$

where $\omega=d \theta$ (therefore $L_{g}$ is a $d \theta$ symmetry $^{40}$ ).

This last equation is the condition to which $\Gamma_{\alpha \beta}$ must satisfy, in order that the action of the group $G$ be canonical. It is the condition for the existence of $\Omega_{g}$ in Eq. (4.9), and, using the expression (3.20) for $\omega$, it gives

$$
d\left(\Gamma_{\alpha \beta} g^{\beta} d y^{\alpha}\right)=0,
$$

or, with the use of Eq. (3.33), we can write

$$
L_{g} \Gamma_{\alpha \beta}+\Gamma_{\alpha \gamma} \frac{\partial g^{\gamma}}{\partial y^{\beta}}+\frac{\partial g^{\gamma}}{\partial y^{\alpha}} \Gamma_{\gamma \beta}=0
$$

Between all possible inequivalent choices of canonical formulations of the dynamics provided by the Lie-König theorem of Sec. III, only those satisfying the condition (4.12) will give rise to a canonical action of the group $G$. It may nevertheless appear that a subgroup of $G$ could only be canonically represented; it will be called $G_{c}{ }^{41}$

It may be seen that the condition (4.12) implies the condition (4.7), but not vice versa. Indeed, if we multiply Eq. (4.12) by $h_{i}^{\alpha}$, since $h_{i}^{\alpha} \Gamma_{\alpha \beta}=0$ [see Eq. (3.34)], we have, after some rearrangement,

$$
\Gamma_{\alpha b}\left(L_{g} h_{i}^{b}-Y_{i} g^{b}+h_{j}^{b} Y_{i} g^{j}\right)=0,
$$

which implies Eq. (4.7), since det $\left\|\Gamma_{a b}\right\| \neq 0$.

If the condition (4.12) is satisfied, and since by hypothesis the manifold on which the original equations of motion are defined is simply connected since it is $R^{2 N+n}$, with a global chart of coordinates $\left\{y^{\alpha}\right\}=\left\{y^{a}, t^{i}\right\}$, from Eq. (4.11) we get

$$
i_{L_{g}} \omega=d h_{g},
$$

that is the vector fields $L_{g}$ are globally Hamiltonian, ${ }^{39}$ with $h_{g}=\Omega_{g}-g^{\alpha} U_{\alpha}$. Using Eq. (3.20) for $\omega$, this can be written

$$
\frac{\partial h_{g}}{\partial y^{\alpha}}=\Gamma_{\alpha \beta} g^{\beta},
$$


which shows that the $h_{g}$ are determined by $\Gamma_{\alpha \beta}$, apart from a set of integration constants, a change of which does not modify the cohomology class, to which the $h_{g}$ belong (see in the following of this section). The difference with respect the one-time formulation lies in the fact that $\omega$ is here $n$-fold degenerate, as it is expected by Eq. (3.26). It follows that the correspondence between $L_{g}$ and $h_{g}$ given by Eq. (4.13) is not $1-1 . L_{g}$ will be determined by $h_{g}$, apart from elements of the kernel distribution of $\omega$, spanned by the vector fields $Y_{i}$.

If we evaluate Eq. (4.13) on the vector field $Y_{i}$, we get

$$
\left(i_{L_{g}} \omega\right)\left(Y_{i}\right)=Y_{i} h_{g},
$$

and, with Eq. (3.26)

$$
Y_{i} h_{g}=0 \text {, }
$$

that is the Hamiltonians $h_{g}$ are constants of motion. This is the ( $n$-time) Noether theorem in the present context. Moreover, by again operating with $i_{L_{g}}$ on Eq. (4.13), we get

$$
L_{g} h_{g}=\mathscr{L}_{L_{g}} h_{g}=0,
$$

i.e., $h_{g}$ is invariant under the action of $L_{g}$.

In order to clarify the role of the vector fields $Y_{i}$, let us consider those infinitesimal transformations (4.3), which leave invariant not only the equations of motion, but even the solutions themselves. They are of the form

$$
y^{\alpha} \rightarrow y^{\alpha}=y^{\alpha}+\epsilon F^{i}(y) h_{i}^{\alpha},
$$

where the $F^{i}(y)$ are $n$ arbitrary functions of all the variables. Indeed, if we require that a particular solution of the equations of motion

$$
y^{\alpha}=f^{\alpha}\left(t^{i}\right) \text {, }
$$

where the integration constants belong to the functional form of $f^{\alpha}$, be left invariant under a transformation (4.3), that is

$$
y^{\prime \alpha}=f^{\alpha}\left(t^{\prime i}\right)
$$

we get for $g^{\alpha}$

$$
g^{\alpha}=h_{i}^{\alpha} g^{i}
$$

with $g^{i}$ arbitrary functions of $y^{\alpha}$.

The transformations (4.16) are canonical transformations, since they satisfy the Eq. (4.13), with $h_{g}=0$, so they belong to the subgroup $G_{c}$, and form a subgroup $K$ of $G_{c}$. The Lie algebra of $K$ is clearly spanned by the vector fields $Y_{i}$, since the corresponding generators $L_{g}$ are given by

$$
L_{F h}=F^{i} h_{i}^{\alpha} \frac{\partial}{\partial y^{\alpha}}=F^{i} Y_{i} .
$$
(4.4).

It easily seen that $K$ is a normal subgroup, due to Eq.

The present situation is again parallel to that discussed in Ref. 22.

Since $K$ is normal, we may consider the factor group $G_{c} / K$, so getting a decomposition of $G_{c}$ (or $G$ ) in cosets, each element of a given coset having the same effect on the solutions of the equations of motion of the others. As in Ref. 22 , in each coset of this decomposition we have only one transformation leaving the times $t^{i}$ fixed. Indeed, if $g_{1}^{\alpha}$ is a given transformation of a coset, by performing a transformation of the same coset such that

$$
F^{i}(y)=-g_{1}^{i}(y),
$$

we get, from Eq. (4.3), with $\alpha=i=1,2, \ldots, n$,

$$
t^{i} \rightarrow\left(t^{\prime}\right)^{i}=t^{i}+\epsilon\left(g_{1}^{i}+F^{i}\right)=t^{i} .
$$

The transformation (4.18) is uniquely determined, so we have in each coset one and only one transformation, which leave all the times fixed. With this choice of the representative of each coset, we have a faithful representation of the group $G_{c} / K$ (or $G / K$ ).

Observe that $\mathrm{Eq}$. (4.12) is satisfied for any $L_{F h}$.

Coming back to Eq. (4.13), let us see what it implies for $L_{g}$, in terms of the canonical variables. As already stressed, the correspondence between $L_{g}$ and $h_{g}$ is not $1-1$, due to the degeneracy of the two-form $\omega$. Equation (4.14) determines $h_{g}$, once the $\Gamma_{\alpha \beta}$ is given. Vice versa, from Eq. (4.14), with $\alpha=a$, and saturated with

$$
\left\{y^{c}, y^{a}\right\}=-\left(\Gamma^{-1}\right)^{c a},
$$

[see Eq. (3.65)], and with Eq. (3.67), we get

$$
\left\{h_{g}, y^{c}\right\}=g^{c}-g^{i} h_{i}^{c} .
$$

Therefore we have the following expression for $L_{g}$ :

$$
L_{g}=g^{\alpha} \frac{\partial}{\partial y^{\alpha}}=\left(g^{\alpha}-g^{i} h_{i}^{a}\right) \frac{\partial}{\partial y^{\alpha}}+g^{i} Y_{i},
$$

that is

$$
L_{g}=\left\{h_{g}, \cdot\right\}+g^{i} Y_{i},
$$

where the $g^{\alpha}$ are such that $i_{L_{g}} \omega=d h_{g}$, for a given $h_{g}$. Let us stress that $g^{i}+\left(g^{\prime}\right)^{i}$ is still solution of this equation, therefore: for given $h_{g}$, the $g^{i}$ remain arbitrary. In Eq. (4.20) the $\{$,$\} is defined as in Eq. (3.61).$

We see from this equation that $L_{g}$ is determined by $h_{g}$, apart from elements of the algebra of the normal subgroup $K$, as

$$
g^{i} Y_{i}=\left(g^{i} h_{i}^{\alpha}\right) \frac{\partial}{\partial y^{\alpha}} .
$$

As a by-product of Eq. (4.13) we also get

$$
\begin{aligned}
& i_{Y_{i}}\left(i_{L_{g}} \omega\right)=i_{Y_{i}} d h_{g}=Y_{i} h_{g}, \\
& -i_{L_{g}}\left(i_{Y_{i}} \omega\right)=Y_{i} h_{g},
\end{aligned}
$$

that is, by using Eq. (3.26)

$$
Y_{i} h_{g}=0 \text {, }
$$

which is another way to get the $n$-time Noether theorem.

We see from Eq. (4.20) that $L_{g}$ is a true canonical generator for those transformations which leave the time fixed, that is when $g^{i}=0$. These transformations are the ones which belong to the faithful representation mentioned before.

From Eq. (4.20) we get an important result. If we consider the commutator

$$
\left[L_{m}-g_{m}^{i} Y_{i}, L_{n}-g_{n}^{j} Y_{j}\right],
$$

where $L_{m}, L_{n}$ are the elements of a basis of the Lie algebra of $G_{c}$, with structure constants $c_{m n}^{k}$, as in Eq. (4.8), if we put

$$
L_{m}=g_{m}^{\alpha} \frac{\partial}{\partial y^{\alpha}}
$$

we find 


$$
\left[L_{m}-g_{m}^{i} Y_{i}, L_{n}-g_{n}^{j} Y_{j}\right]=c_{m n}^{k}\left(L_{k}-g_{k}^{i} Y_{i}\right),
$$

where we have used Eq. (4.6).

The result (4.22) implies for $h_{g}$ :

$$
\left\{h_{m}, h_{n}\right\}=c_{m n}^{k} h_{k}+d_{m n},
$$

where the quantities $d_{m n}$ are constants. They are constant in $t^{i}$ even, as the $h_{m}$ are constants of motion, and their Poisson bracket too. They must also satisfy the following relations:

$$
\begin{aligned}
& d_{m n}=-d_{n m}, \\
& c_{m n}^{k} d_{k l}+c_{n l}^{k} d_{k m}+c_{l m}^{k} d_{k n}=0,
\end{aligned}
$$

that is the $d_{m n}$ are the components of a two-cocycle defined on the Lie algebra $\mathscr{G}_{c}$ of $G_{c}$.

Let us once again stress that the explicit expressions for the $h_{g}$ are determined by the choice of the $\Gamma_{\alpha \beta}$, as shown by Eq. (4.14), apart from the choice of some integration constants (in all the variables $y^{a}$ and $t^{i}$ ). Different choices of these integration constants will give different Hamiltonians $h_{g}$, belonging to the same two-cocycle class of the two-cohomology group $H^{2}(\mathscr{G})$ of $G^{42}$ Only if $H^{2}(\mathscr{G})=0$, will in general be possible to make a choice that eliminates the quantities $d_{m n}$, that is when the $d_{m n}$ are of the form $c_{m n}^{k} c_{k} \cdot{ }^{43}$

Another point which is worth mentioning, connected with the previous discussion, is the existence of transformations which leave the action invariant ${ }^{44}$ that is such that

$$
\theta \stackrel{L_{g}}{\rightarrow} \theta^{\prime}=\theta, \quad d \Omega_{g}=0 .
$$

Again following Ref. 22 we will look for a canonical transformation

$$
\theta \rightarrow \theta^{\prime}=\theta+d \Lambda,
$$

such that, combined with the transformation (4.9) generated by $G$, it will give a transformation leaving $\theta$ invariant, that is such that

$$
\mathscr{L}_{L_{g}} \theta^{\prime}=\mathscr{L}_{L_{g}}(\theta+d \Lambda)=d\left(\Omega_{g}+L_{g}(\Lambda)\right)=0 .
$$

If we can choose

$$
L_{g}(\Lambda)=-\Omega_{g},
$$

we will have $\theta$ invariant.

The condition for the existence of a function $\Lambda$ such that

$$
\mathscr{L}_{L_{s}}(\theta+d \Lambda)=0
$$

can be obtained as follows. From Eq. (4.28) we get

$$
d\left(h_{g}+i_{L_{g}} \theta+\mathscr{L}_{L_{g}} \Lambda\right)=0,
$$

where we used Eq. (4.13), that is

$$
\mathscr{L}_{L_{\mathrm{g}}} \Lambda=-\left(h_{g}+i_{L_{\mathrm{g}}} \theta\right),
$$

apart from a constant, which can be reabsorbed in $h_{g}$.

If $\left\{L_{m}\right\}$ is a basis of the Lie algebra $\mathscr{G}$, as in Eq. (4.8), we have from Eq. (4.29)

$$
\begin{aligned}
& {\left[\mathscr{L}_{L_{m}}, \mathscr{L}_{L_{n}}\right]^{\Lambda}} \\
& \quad=-\mathscr{L}_{L_{m}}\left(h_{n}+i_{L_{n}} \theta\right)+\mathscr{L}_{L_{n}}\left(h_{m}+i_{L_{m}} \theta\right),
\end{aligned}
$$

or, since

$$
\left[\mathscr{L}_{L_{m}}, \mathscr{L}_{L_{n}}\right]=\mathscr{L}_{\left[L_{m} L_{n}\right]}=c_{m n}^{k} \mathscr{L}_{L_{k}},
$$

$$
\begin{aligned}
c_{m n}^{k} & \left(-h_{k}-i_{L_{k}} \theta\right) \\
& =-\mathscr{L}_{L_{m}}\left(h_{n}+i_{L_{n}} \theta\right)+\mathscr{L}_{L_{n}}\left(h_{m}+i_{L_{m}} \theta\right) .
\end{aligned}
$$

Now we have

$$
\begin{aligned}
c_{m n}^{k} h_{k} & -\mathscr{L}_{L_{m}} h_{n}+\mathscr{L}_{L_{n}} h_{m} \\
& =c_{m n}^{k} h_{k}-L_{m}\left(h_{n}\right)+L_{n}\left(h_{m}\right) \\
& =c_{m n}^{k} h_{k}-\left\{h_{m}, h_{n}\right\}+\left\{h_{n}, h_{m}\right\} \\
& =c_{m n}^{k} h_{k}-2\left\{h_{m}, h_{n}\right\},
\end{aligned}
$$

since, from Eqs. (4.20) and (4.15), we have

$$
L_{m}\left(h_{n}\right)=\left\{h_{m}, h_{n}\right\} \text {. }
$$

On the other hand, since

$$
i_{[X, Y]}=\mathscr{L}_{X^{i_{Y}}}-i_{Y} \mathscr{L}_{X},
$$

we have

$$
\begin{aligned}
& \left(\mathscr{L}_{L_{L_{m}}} i_{L_{n}}-\mathscr{L}_{L_{n}} i_{L_{m}}\right) \theta \\
& \quad=i_{\left[L_{m} L_{n}\right]} \theta-i_{L_{m}} i_{L_{n}} \omega \\
& =c_{m n}^{k} i_{L_{k}} \theta-\left\{h_{m}, h_{n}\right\},
\end{aligned}
$$

where we used the fact that for any one-form $\theta$ it holds

$$
\mathscr{L}_{X} i_{Y} \theta=i_{X} \mathscr{L}_{Y} \theta-i_{X} i_{Y} d \theta,
$$

and that

$$
i_{L_{m}} i_{L_{n}} \omega=i_{L_{m}} d h_{n}=L_{m}\left(h_{n}\right)=\left\{h_{m}, h_{n}\right\} .
$$

Collecting these results we get that the condition (4.30) for the existence of $\Lambda$ becomes

$$
c_{m n}^{k} h_{k}-\left\{h_{m}, h_{n}\right\}=0 \text {. }
$$

$\Lambda$ will exist when this relation holds for the $h_{m}$, or for the $h_{m}$ with some constant $c_{m}$ added, since in Eq. (4.29) we disposed of an additive constant for each $h_{m}$. This means that the $h_{m}$ must belong to a two-cohomology class equivalent to zero, or that it must be $H^{2}(\mathscr{G})=0$.

When the condition (4.33) holds, the group $G$ is canonically realized and the $h_{m}$ are comoments ${ }^{34}$; we know that this is always possible for the inhomogeneous Lorentz group, but not for the Galilei group.

In the $n$-time canonical formalism the dynamics is described in terms of variables which are functions of $n$ independent times, that is the $\left\{\mathbf{x}^{r}\right\}$ and the $\left\{\mathbf{p}_{i}\right\}$; at equal times we should recover the usual canonical formalism. This implies that we should require that the canonical coordinates $\left\{x^{i}\right\}$ and the position coordinates $\left\{\mathbf{q}^{i}\right\}$ be coinciding at equal times [see Eq. (3.75) ]. It is just the condition which defines the position coordinates in a unique way, when we follow the reverse procedure. This definition of the position coordinates is due to Droz-Vincent, ${ }^{20}$ who gave it in a relativistic context. The reverse procedure was used by him in order to build explicit models for two relativistic bodies.

In Sec. II and III we started from a set of equations of motion, written in a form best suited for the nonrelativistic dynamics. However, all that we have said is quite general, and not restricted to the nonrelativistic case.

In the next section we will develop the before mentioned reverse procedure, and we will discuss the position coordinates problem. 


\section{EXTENDED PHASE SPACE AND CONSTRAINED DYNAMICS}

In order to develop an a priori canonical theory, from which to recover the position coordinates and their equations of motion, and besides to make contact with the constraint's theory, ${ }^{6}$ it is useful to introduce an extended phase space, by defining a set of $n$ new coordinates conjugated to the $n$ times $t^{i}$, which will be called the energies $\epsilon_{i}$, with

$$
\left\{\epsilon_{i}, t^{j}\right\}=\delta_{i}^{i} \text {. }
$$

The new Poisson brackets will be

$$
\{A, B\}^{\prime}=\{A, B\}+\frac{\partial A}{\partial \epsilon_{i}} \frac{\partial B}{\partial t^{i}}-\frac{\partial B}{\partial \epsilon_{i}} \frac{\partial A}{\partial t^{i}} .
$$

The space of the variables $\left\{\mathbf{x}^{i}, \mathbf{p}_{i}, t^{i}\right\}$ of the previous sections is recovered by requiring the constraints

$$
\psi_{i}=0
$$

with

$$
\psi_{i}=\epsilon_{i}-H_{i}(\mathbf{x}, \mathbf{p}, t)
$$

Since the $H_{i}$ satisfy the integrability conditions (3.60), we have that the functions $\psi_{i}$ are first class constraints ${ }^{6}$

$$
\left\{\psi_{i}, \psi_{j}\right\}^{\prime}=0 \text {. }
$$

The manifold $M$, defined by Eq. (5.2), which is the original space of the previous section, now becomes a submanifold of the new phase space $\bar{M}=R^{2(N+n)}(N=3 n)$, since the rank of the matrix $\left\|\partial \psi_{i} / \partial(x, p, t, \epsilon)\right\|$ is clearly $n$ over all $M$, and this guarantees that $M$ is a $2 N+n$ submanifold of $\bar{M}{ }^{45}$

The manifold $\bar{M}$ can be defined as a symplectic manifold $(\bar{M}, \bar{\omega})$, with a two-form $\bar{\omega}$ defined by

$$
\begin{aligned}
& \bar{\omega}=d \bar{\theta}, \\
& \bar{\theta}=\mathbf{p}_{i} d \mathbf{x}^{i}-\epsilon_{i} d t^{i},
\end{aligned}
$$

which is clearly a closed nondegenerate two-form.

The submanifold $M$ with the two-form $\omega=d \theta$, defined in Eq. (3.62), which is closed but degenerate [see Eq. (3.26) ], is a presymplectic manifold, in which a preferred Poisson structure has been introduced with Eq. $(3.61) .^{28}$ The definition of $M$ as a submanifold of $\bar{M}$ is an example of a theorem demonstrated by Gotay, ${ }^{27}$ which asserts that every presymplectic manifold may be coisotropically embedded in a symplectic manifold. This means that $M$ is a coisotropic submanifold of $\bar{M}$, which, in the language of the constraint's theory, means that it is defined by a set of first class constraints, exactly as we have seen with Eq. (5.4).

The conditions that must be satisfied for a coisotropic embedding are expressed in the following way. Let us define the mapping

$$
j: M \rightarrow \bar{M},
$$

as specified by the conditions (5.2). The conditions are now

(i) $\omega=j^{*} \bar{\omega}$,

(ii) $T M^{\perp} \subseteq T_{j}(T M)$,

where $T M(T \bar{M})$ is the set of tangent vectors on $M(\bar{M})$, $T_{j}(T M)$ is the set of tangent vectors on $\bar{M}$, which are tangent to the submanifold $M$, and $T M^{\perp}$ is defined as the set

$$
T M^{\perp}=\left\{\bar{X} \in T \bar{M}, \bar{\omega}(\bar{X}, \bar{Y})=0, \forall \bar{Y} \in T_{j}(T M)\right\},
$$

called the $\bar{\omega}$-orthogonal complement of $M^{34}$

We now verify that the conditions (i) and (ii) are satisfied, and, in doing that, we will get the precise connection between the extended phase space $\bar{M}$ and the original space $\boldsymbol{M}$.

It is immediately seen from the definition of $\bar{\omega}$ in Eq. (5.5) and of $\omega$ in Eqs. (3.62) and (3.20), that the condition (i) is satisfied. Indeed we have that the mapping $j$ is defined by the transformation

$$
j:\left(\mathbf{x}^{i i}, \mathbf{p}^{\prime}, t^{\prime i}\right) \rightarrow\left(\mathbf{x}^{i}, \mathbf{p}_{i}, t^{i}, \epsilon_{i}\right),
$$

with

$$
\begin{aligned}
& \mathbf{x}^{i}=\mathbf{x}^{i}, \quad \mathbf{p}_{i}=\mathbf{p}_{i}^{\prime}, \quad t^{i}=t^{\prime i}, \\
& \epsilon_{i}=H_{i}\left(\mathbf{x}^{i}, \mathbf{p}_{i}, t^{i}\right)
\end{aligned}
$$

so we have

$$
\begin{aligned}
j^{*} \bar{\omega} & =j^{*}\left(d \mathbf{p}_{i} \wedge d \mathbf{x}^{i}-d \epsilon_{i} \wedge d t^{i}\right) \\
& =d \mathbf{p}_{i} \wedge d \mathbf{x}^{i}-d H_{i}(\mathbf{x}, \mathbf{p}, t) \wedge d t^{i}=\omega .
\end{aligned}
$$

On the other hand, given any vector field on $M$

$$
X=\mathbf{a}^{i} \cdot \frac{\partial}{\partial \mathbf{x}^{i}}+\mathbf{b}_{i} \cdot \frac{\partial}{\partial \mathbf{p}_{i}}+c^{i} \frac{\partial}{\partial t^{i}},
$$

we have on $\bar{M}$

$$
\begin{aligned}
\bar{X}= & T_{j}(X)=\mathbf{a}^{i} \cdot\left(\frac{\partial}{\partial \mathbf{x}^{i}}+\frac{\partial H_{j}}{\partial \mathbf{x}^{i}} \frac{\partial}{\partial \epsilon_{j}}\right) \\
& +\mathbf{b}_{i} \cdot\left(\frac{\partial}{\partial \mathbf{p}_{i}}+\frac{\partial H_{j}}{\partial \mathbf{p}_{i}} \frac{\partial}{\partial \epsilon_{j}}\right) \\
& +c^{i}\left(\frac{\partial}{\partial t^{i}}+\frac{\partial H_{j}}{\partial t^{i}} \frac{\partial}{\partial \epsilon_{j}}\right)
\end{aligned}
$$

where the functions $\mathbf{a}^{i}, \mathbf{b}^{i}$, and $c^{i}$ are restricted on $M$; this is the form of a vector field on $\bar{M}$ tangent to $M$. A vector field $\bar{X}$ of $\bar{M}$ belonging to $T M^{1}$ is such that

$$
\bar{\omega}=(\bar{X}, \bar{Y})=0, \text { for any } \bar{Y} \in T_{j}(T M),
$$

and if $\bar{X}$ is given in components as

$$
\bar{X}=\alpha^{i} \cdot \frac{\partial}{\partial \mathbf{x}^{i}}+\beta_{i} \cdot \frac{\partial}{\partial \mathrm{p}_{i}}+\gamma^{i} \frac{\partial}{\partial t^{i}}+\delta_{i} \frac{\partial}{\partial \epsilon_{i}},
$$

this means

$$
\begin{aligned}
& \alpha^{i}=\frac{\partial H_{j}}{\partial \mathrm{p}_{i}} \gamma^{j}, \quad \beta_{i}=-\frac{\partial H_{j}}{\partial \mathrm{x}^{i}} \gamma^{i}, \\
& \delta_{i}=-\frac{\partial H_{j}}{\partial t^{i}} \gamma^{j}
\end{aligned}
$$

Substituting in Eq. (5.11) we get

$$
\bar{X}=\gamma^{j} \bar{Y}_{j} \text {, }
$$

where

$$
\bar{Y}_{j}=\left\{\psi_{j}, \cdot\right\}^{\prime} \quad(j=1,2, . ., n) .
$$

These $n$ vector fields are precisely the images under $j$ of the fields $Y_{i}$, annihilating the two-form $\omega$

$$
i_{Y_{i}} \omega=0 \text {, }
$$

that is 
$T_{j}\left(Y_{i}\right)=\bar{Y}_{i}+\left(\frac{\partial H_{j}}{\partial t^{i}}-\frac{\partial H_{i}}{\partial t^{j}}+\left\{H_{j}, H_{i}\right\}\right) \frac{\partial}{\partial \epsilon_{j}}=\bar{Y}_{i}$,

due to the integrability conditions for the $H_{i}$.

So we have that $T M^{1}$ is spanned by these vector fields $\bar{Y}_{i}$, which span the kernel distribution of $\omega$, as found in Sec. III.

The coincidence of the kernel distribution of $\omega$ and $T M^{\perp}$ is characteristic of the coisotropic case; in particular, the vector fields $\bar{Y}_{i}$ generate the functions $\psi_{i}$, which define the submanifold $\boldsymbol{M}$

$$
i_{\overline{\mathbf{v}_{i}}} \overline{\boldsymbol{\omega}}=d \psi_{i},
$$

which corresponds to Eq. (5.13).

It is now easily verified that even the condition (ii) is satisfied. Indeed any $\bar{Y} \in T_{j}(T M)$ has the form (5.10), and, with the choice

$$
\mathbf{a}^{i}=\frac{\partial H_{j}}{\partial \mathrm{p}_{i}}, \quad \mathbf{b}_{i}=-\frac{\partial H_{j}}{\partial \mathbf{x}^{i}}, \quad c^{i}=\delta_{j}^{i},
$$

for any given $j$, and making again use of the integrability conditions for the $H_{j}$, we get that Eq. (5.10) becomes Eq. (5.14), which shows that $T M^{1} \subseteq T_{j}(T M)$.

In conclusion we have that the two conditions for a coisotropic embedding are satisfied. The relation between $\bar{M}$ and the submanifold $M$ is given by Eqs. (5.8) and (5.10). The equations of motion, which were given by Eq. (3.71), are now given by Eq. (5.16), with the vector fields $\bar{Y}_{i}$ given by Eqs. (5.14), to which we have to add the algebraic equations $\psi_{i}=0$. It is clear from Eq. (5.4) that the $\psi_{i}$ are constants of motion.

From Eq. (5.16) we get explicitly the equations of motion, as the equations for the integral curves of the vector fields $\bar{Y}_{i}$

$$
\begin{aligned}
d \mathbf{x}^{i} & =\left\{\mathbf{x}^{i}, \psi_{j}\right\}^{\prime} d \tau^{j}, \\
d \mathbf{p}_{i} & =\left\{\mathbf{p}_{i}, \psi_{j}\right\}^{\prime} d \tau^{j}, \\
d t^{i} & =\left\{t^{i}, \psi_{j}\right\}^{\prime} d \tau^{j}, \\
d \epsilon_{i} & =\left\{\epsilon_{i} \psi_{j}\right\}^{\prime} d \tau^{j},
\end{aligned}
$$

where $j=1,2, \ldots, n$.

Due to the particular form of the functions $\psi_{j}$, the equations for the times are simply

$$
d t^{i}=-d \tau^{i},
$$

which determine the parameters $\tau^{j}$ in terms of physical coordinates. In this way, and using the constraints (5.2), we recover the equations of motion (3.73).

Since the manifold $(\bar{M}, \bar{\omega})$ is symplectic, that is the closed two-form $\bar{\omega}$ is now not degenerate, the correspondence between the functions $\psi_{j}$ and the vector fields $\bar{Y}_{j}$ given by Eqs. (5.14) or (5.16) does not have the ambiguity of the analogous corespondence in the space $M$, manifested by the appearance of the undetermined components $g^{i}$ in Eq. (4.19).

This is also true for the Hamiltonian vector fields generating the transformations of the invariance group $G$ of Sec. IV. We have indeed, using Eq. (5.10), that the vector fields on $\bar{M}$ corresponding to the vector fields $L_{g}$ of Sec. IV in Eq. (4.12), are

$$
\bar{L}_{g}=T_{j}\left(L_{g}\right)=\left\{h_{g}, \cdot\right\}^{\prime}+g^{i}\left\{\psi_{i}, \cdot\right\}^{\prime} .
$$

In getting this result we have to use the fact that the $h_{g}$ are constants of motion [see Eq. (4.15)], which can now be written

$$
\left\{h_{g}, \psi_{i}\right\}^{\prime}=0,
$$

and the first class character of the $\psi_{i}$.

If we choose a basis $\left\{h_{m}\right\}$ for the Hamiltonians $h_{g}$, such that

$$
\bar{L}_{m}=\left\{h_{m}, \cdot\right\}^{\prime},
$$

where $\bar{L}_{m}=T_{j}\left(L_{m}\right)$, and where the $L_{m}$ satisfy the commutation relations $(4.8)$, we see, from Eq. $(5.20)$, that any $\bar{L}_{g}$ can be written as

$$
\bar{L}_{g}=\sum_{m} a_{m} \bar{L}_{m}+\sum_{i=1}^{n} g^{\bar{Y}_{i}},
$$

which agrees with

$$
i_{\bar{L}_{g}} \bar{\omega}=d \bar{h}_{g} \text {, }
$$

or

$$
\bar{L}_{g}=\left\{\bar{h}_{g}, \cdot\right\}^{\prime},
$$

where

$$
d \bar{h}_{g}=\sum_{m} a_{m} d h_{m}+\sum_{i=1}^{n} g^{i} d \psi_{i} .
$$

Equation (5.24) is easily verified

$$
\begin{aligned}
i_{\bar{L}_{g}} \bar{\omega} & =\sum_{m} a_{m} i_{\bar{L}_{m}} \bar{\omega}+\sum_{i=1}^{n} g^{i} i_{\bar{Y}_{i}} \bar{\omega} \\
& =\sum_{m} a_{m} d h_{m}+\sum_{i=1}^{n} g^{i} d \psi_{i}=d \bar{h}_{g} .
\end{aligned}
$$

From Eq. (5.24) we also get

$\bar{L}_{g}=\left\{\bar{h}_{g}, \cdot\right\}^{\prime}=\sum_{m} a_{m}\left\{h_{m}, \cdot\right\}^{\prime}+\sum_{i=1}^{n} g^{i}\left\{\psi_{i}, \cdot\right\}^{\prime}$.

The vector fields $\bar{L}_{m}$ and $\bar{Y}_{i}$, tangent to the submanifold $M$, leave invariant the constraints hypersurface $\psi_{i}=0$, indeed

$$
\begin{aligned}
& \bar{L}_{m} \psi_{i}=\left\{h_{m}, \psi_{i}\right\}^{\prime}=0, \\
& \bar{Y}_{j} \psi_{i}=\left\{\psi_{j}, \psi_{i}\right\}^{\prime}=0,
\end{aligned}
$$

where the first of these equations comes from the constancy of the Hamiltonians $h_{m}$ [see Eq. (5.21)], and the second corresponds to Eq. (5.4).

In other words, Eqs. (5.28) show that under the action of the algebra $\mathscr{G}$ of the invariance group $G$, of which the vector fields $\bar{Y}_{i}$ are an Abelian subalgebra, the new energy canonical coordinates $\epsilon_{i}$ are defined in such a way to transform like the Hamiltonians $\boldsymbol{H}_{i}$

$$
\begin{aligned}
& \left\{h_{m}, \epsilon_{i}\right\}^{\prime}=\left\{h_{m}, H_{i}\right\}^{\prime}, \\
& \left\{\psi_{j}, \epsilon_{i}\right\}^{\prime}=\left\{\psi_{j}, H_{i}\right\}^{\prime} .
\end{aligned}
$$

The meaning of the subalgebra spanned by the vector fields $\bar{Y}_{i}$ is that it generates the transformations of reparametrization $\tau^{i} \rightarrow \tau^{\prime i}=\phi^{i}(\tau)$ of the equations of motion (5.18), and are invariances of the dynamics. 
Observe however that the canonical coordinates $\left\{\mathbf{x}^{i}, \mathbf{p}_{i}, t^{i}, \epsilon_{i}\right\}$ are not observables, in the usual meaning in which this word is used in the constraints theory ${ }^{46}$; the observables, that is the canonical quantities with zero Poisson brackets with the constraints $\psi_{i}$ are more precisely the constants of motion (that is the independent initial data of the dynamical problem). This is a slight departure from the usual gauge theories.

The systems under consideration have vanishing canonical Hamiltonian, $H_{c}=0$, and are described by $n$ firstclass constraints $\psi_{i}=0$, which are in strong involution [see Eq. (5.4) ], and which are generators of the gauge transformations in $\bar{M}$.

In the standard approach ${ }^{6}$ one introduces the Dirac Hamiltonian

$$
H_{D}=\sum_{i=1}^{n} \lambda^{i}(\tau) \psi_{i}
$$

and one writes the following Hamiltonian equations:

$$
\frac{d A(\tau)}{d \tau}=\left\{A(\tau), H_{D}\right\}^{\prime} \approx \sum_{i} \lambda^{i}(\tau)\left\{A(\tau), \psi_{i}\right\}^{\prime},
$$

where $A$ is any of the canonical variables of the extended phase space, function of a scalar parameter $\tau$. These equations describe the most general gauge transformations in $\bar{M}$, due to the arbitrariness of the Lagrange multipliers $\lambda^{i}(\tau)$. Equations (5.18) are recovered by introducing $n$ independent parameters $\tau^{i}$ in the following way:

$$
d \tau^{i}=\lambda^{i}(\tau) d \tau,
$$

and by a redefinition of the quantity $A$ as function of the $n \tau^{i}$.

We now have all the ingredients for a discussion of the formalism in the extended phase space.

Here we are most interested in discussing the problem of the physical positions $\mathbf{q}^{i}$, when we start with an a priori canonical dynamics. Following Ref. 20, the position coordinates can be defined as the solutions of the following partial differential equations of the first order:

$$
\bar{Y}_{j} \mathbf{q}^{i}=-\left\{\mathbf{q}^{i}, \psi_{j}\right\}^{\prime}=0, \text { for } i \neq j,
$$

where $i j=1,2, \ldots, n$, and with

$$
\mathbf{q}^{i}=\mathbf{x}^{i}, \quad \text { at } t^{i}=t,
$$

as Cauchy surface for the definition of the initial conditions.

Observe that the hypersurface $t^{i}=t$ in the phase space is not invariant under the action of the vector fields $\bar{Y}_{i}$. This is the required condition for the Lemma (V9) of Ref. 20 to hold. The $q^{i}$ determined by Eqs. (5.33) and (5.34) are unique. They are a function of the $\tau^{i}$, but, in view of Eq. (5.19), they can be thought of as a function of the times $t^{i}$.

From Eq. (5.33) it follows

$$
\begin{aligned}
& \mathbf{v}^{i}=-\left\{\mathbf{q}^{i}, \psi_{i}\right\}^{\prime}=\bar{Y}_{i} \mathbf{q}^{i}, \\
& \mathbf{a}^{i}=\left\{\left\{\mathbf{q}^{i}, \psi_{i}\right\}^{\prime}, \psi_{i}\right\}^{\prime}=\bar{Y}_{i} \mathbf{v}^{i},
\end{aligned}
$$

and

$$
\begin{array}{ll}
\bar{Y}_{j} \mathbf{v}^{i}=-\left\{\mathbf{v}^{i}, \psi_{j}\right\}^{\prime}=0, & \text { for } i \neq j, \\
\bar{Y}_{j} \mathbf{a}^{i}=-\left\{\mathbf{a}^{i}, \psi_{j}\right\}^{\prime}=0, & \text { for } i \neq j .
\end{array}
$$

Observe that Eqs. (5.21) ensure the invariance of the solutions of Eq. (5.33) under the invariance group $G$.
In the spirit of the present approach, we have to look for position coordinates $\mathbf{q}^{i}$ not depending on the energies $\epsilon_{i}$, since the $q^{i}$ must live in the original phase space. So we can reinforce the requirement (5.33) with

$\left\{\mathbf{q}_{;}^{i} t^{\jmath}\right\}^{\prime}=0$, for any $j$.

From these equations it follows:

$\left\{\mathbf{v}^{i}, t^{\prime}\right\}^{\prime}=0$,

$\left\{\mathbf{a}^{i}, t^{\prime}\right\}^{\prime}=0$.

From Eqs. (5.33), (5.39), (5.40), and (5.41) it follows:

$\mathbf{v}^{i}=\bar{Y}_{i} \mathbf{q}^{i}=Y_{i} \mathbf{q}^{i}$,

$\mathbf{a}^{i}=\bar{Y}_{i} \mathbf{v}^{i}=Y_{i} \mathbf{v}^{i}$,

where $Y_{i}$ are the vector fields on $M$ defined in Eq. (3.70).

In the case in which the restricted integrability conditions (3.1) hold, the $q^{i}$ and the $v^{i}$ coincide with the original $y^{a}$ of Eq. (3.3). Actually, we have recovered Eqs. (2.9), starting from a phase-space approach. As a by-product, the variables $\mathbf{q}^{i}$ so obtained have the same covariance properties of the original $n$-time physical coordinates, and, like them, are not canonical variables. Of course, they become canonical variables when at least one Lagrangian does exist for the Newtonian system [see Eq. (3.76)].

Also in the $n$-time formulation of the nonrelativistic theory one of the forms of the no-interaction theorem of the relativistic dynamics ${ }^{18}$ has been obtained: the identification $\mathbf{q}^{i}=\mathbf{x}^{i}$, i.e., both covariant and canonical, is allowed in the free case only, because only then it is $\mathrm{x}^{i}\left(t^{1}, t^{2}, \ldots, t_{n}\right)$ $=\mathbf{x}^{i}\left(t^{i}\right)=\mathbf{q}^{i}\left(t^{i}\right)$ (see Ref. 47 for a nonrelativistic no-interaction theorem).

We now give a formal demonstration of the nonrelativistic $n$-time no-interaction theorem (which can however be also applied to a relativistic system described by a set of firstclass constraints). In this demonstration no use is done of the canonical kinematical Galilei (Poincaré) algebra (see the review paper quoted in Ref. 18 for a comparison).

Our hypotheses are that the system is described by the first-class constraints (5.2) in $\bar{M}$; the physical coordinates $\mathbf{q}^{i}$ are obtained from Eqs. (5.33) and (5.34), with the $\mathbf{v}^{i}$ and $\mathbf{a}^{i}$ given by Eqs. (5.35) and (5.36), and moreover Eq. (5.34) still holds when the times are different, so that

$$
\left\{q^{i, m}, q^{i, k}\right\}^{\prime}=\left\{x^{i, m}, x^{i, k}\right\}^{\prime}=0
$$

[ this is the crucial hypothesis, since it implies $\mathbf{x}^{i}=\mathbf{x}^{i}\left(t^{i}\right)$ ].

A final hypothesis, which is also needed in the standard relativistic formulation, is that

$$
\operatorname{det}\left(\frac{\partial v^{i, m}}{\partial p_{i, k}}\right) \neq 0
$$

From Eq. (5.33) and from $\left\{q^{i, m}, q^{j, k}\right\}^{\prime}=0$ we get

$$
\left\{q^{i, m}, v^{j, k}\right\}^{\prime}=0 \text {, for } i \neq j \text {, }
$$

which implies

$$
\frac{\partial v^{j, k}}{\partial p_{i, m}}=0, \text { for } i \neq j .
$$

Then we get 


$$
\operatorname{det}\left(\frac{\partial v^{j, k}}{\partial p_{i, m}}\right)=\prod_{i} \operatorname{det}\left(\frac{\partial v^{i, k}}{\partial p_{i, m}}\right) \neq 0,
$$

due to Eq. (5.43).

From Eqs. (5.4), (5.33), and (5.44) we obtain

$$
\begin{aligned}
& \left\{v^{i, m}, \psi_{j}\right\}^{\prime}=0, \text { for } i \neq j, \\
& \left\{a^{i, m}, \psi_{j}\right\}^{\prime}=0 \text {, for } i \neq j \text {, } \\
& \left\{v^{i, m}, v^{j, k}\right\}^{\prime}=0 \text {, for } i \neq j \text {, } \\
& \left\{q^{i, m}, a^{i, k}\right\}^{\prime}=0, \text { for } i \neq j .
\end{aligned}
$$

From the last of these equations, and with the use of Eq. (5.44), we get

$\frac{\partial a^{j, k}}{\partial p_{i, m}}=\sum_{l} \frac{\partial \mathbf{v}^{l}}{\partial p_{i, m}} \cdot \frac{\partial a^{j, k}}{\partial \mathbf{v}^{l}}=\frac{\partial \mathbf{v}^{i}}{\partial p_{i, m}} \cdot \frac{\partial a^{j, k}}{\partial \mathbf{v}^{i}}=0$, for $i \neq j$.

Equation (5.43) then implies

$$
\frac{\partial a^{i, k}}{\partial v^{i, m}}=0, \text { for } i \neq j
$$
imply

Finally, Eqs. (5.37), (5.44) and the third of Eq. (5.46)

$$
\begin{aligned}
\left\{v^{i, m}, a^{j, k}\right\}^{\prime} & =-\sum_{i} \frac{\partial v^{i, m}}{\partial \mathbf{p}_{l}} \cdot \frac{\partial a^{j, k}}{\partial \mathbf{q}^{l}} \\
& =-\frac{\partial v^{i, m}}{\partial \mathbf{p}_{i}} \cdot \frac{\partial a^{j, k}}{\partial \mathbf{q}^{i}}=0, \text { for } i \neq j,
\end{aligned}
$$

and, from Eq. (5.43)

$$
\frac{\partial a^{j, k}}{\partial q^{i, m}}=0, \text { for } i \neq j
$$

Equations (5.47) and (5.49) give the result

$$
\mathbf{a}^{i}=\mathbf{a}^{i}\left(\mathbf{q}^{i}, \mathbf{v}^{i}, t^{i}\right) \text {. }
$$

Space-time invariance then implies

$$
\mathbf{a}^{i}=\mathbf{a}^{i}\left(\mathbf{v}^{i}\right) \text {, }
$$

and this equation describes a free motion only.

In the case of the restricted integrability conditions (3.1), and by looking at the one-form $\theta$ [see Eqs. (3.10) and (3.62)]

$$
\begin{aligned}
S=\int_{a(l)}^{b} \theta & =\int_{a(l)}^{b}\left(\mathbf{U}_{i} \cdot d \mathbf{q}^{i}+\mathbf{U}_{i+n} \cdot d \mathbf{v}^{i}-V_{i} d t^{i}\right) \\
& =\int_{a(l)}^{b}\left(\mathbf{p}_{i} \cdot d \mathbf{x}^{i}-H_{i} d t^{i}\right)
\end{aligned}
$$

we see that the requirement $\mathrm{q}^{i}=\mathbf{x}^{i}$ at different times, leading to the no-interaction theorem, implies

$$
\begin{aligned}
& \mathbf{U}_{i}=\mathbf{p}_{i}, \\
& \mathbf{U}_{i+n}=\frac{\partial \phi\left(\mathbf{v}^{1}, \mathbf{v}^{2}, \ldots, \mathbf{v}^{n}\right)}{\partial \mathbf{v}^{i}}, \\
& V_{i}=H_{i} .
\end{aligned}
$$

Therefore, $\mathbf{U}_{i+n} \cdot d \mathbf{v}^{i}=d \phi\left(\mathbf{v}^{1}, \mathbf{v}^{2}, \ldots, \mathbf{v}^{n}\right)$, and this surface term can be eliminated from the action (3.10). Moreover, the form of $U_{i+n}$ implies

$$
\Gamma_{i+n, m ; j+n, k}=0 \Rightarrow \Gamma_{a b}=\left(\begin{array}{cc}
a & b \\
-b & 0
\end{array}\right)
$$

at different times. Vice versa Eq. (5.52) implies the second of Eqs. (5.51), and Eq. (5.50) gives

$$
\mathrm{U}_{i} \cdot d \mathbf{q}^{i}-V_{i} d t^{i}+d \phi=\mathbf{p}_{i} \cdot d \mathbf{x}^{i}-H_{i} d t^{i} .
$$

This last equation can be rewritten

$$
\begin{aligned}
U_{i} \cdot d \mathbf{q}^{i}-V_{i} d t^{i} & =\mathbf{p}_{i} \cdot d \mathbf{x}^{i}-H_{i} d t^{i}-d \phi \\
& =\mathbf{p}_{i}^{\prime} \cdot d \mathbf{x}^{i}-H^{\prime}{ }_{i} d t^{i},
\end{aligned}
$$

where $\mathbf{x}^{i}, \mathbf{p}_{i}{ }_{i}, H^{\prime}{ }_{i}$ are defined by the canonical transformation associated to $\phi$. Therefore Eq. (5.42) imply

$$
\mathbf{q}^{i}=\mathbf{x}^{\prime i}, \quad \mathbf{U}_{i}=\mathbf{p}_{i}^{\prime}, \quad V_{i}=H^{\prime}{ }_{i},
$$

at different times.

This is a new formulation of the no-interaction theorem: if $\Gamma_{a b}$ satisfies Eq. (5.52) at different times, a free motion only is allowed.

Let us return to the line action (3.10) for the first-order system (2.9). A necessary and sufficient condition ${ }^{48}$ to get from Eq. (3.10) an action for a second-order system, independent of the accelerations, is

$$
\mathbf{U}_{i+n}=\frac{\partial \phi\left(\mathbf{v}^{1}, \mathbf{v}^{2}, \ldots, \mathbf{v}^{n}\right)}{\partial \mathbf{v}^{i}},
$$

where

$$
\mathbf{v}^{i}=\frac{d \mathbf{q}^{i}}{d t}
$$

But, as we have just seen, this condition implies the no-interaction theorem. Therefore we get the result that an acceleration independent action for the $\boldsymbol{n}$-time second-order equation (2.6) (which could be called a predictive action) does not exist, except in the free case. As shown in Ref. 49 a predictive action, giving rise to a canonical realization of the kinematical algebra at the Galilei or Poincaré groups, can be only obtained with a Fokker-like action, depending on the accelerations of every order. Instead, if we restrict the line action (3.10) to the path $t^{1}=t^{2}=\cdots=t^{n}$, the previous condition on $\mathbf{U}_{i+n}$ implies Eq. (5.52) at equal times only, and this is Eq. (3.76), which was shown to be the condition for the existence of a Lagrangian associated to the canonical structure $\Gamma_{a b}$. Let us remark that, if the canonical structure $\Gamma_{a b}$ does not admit a Lagrangian, the corresponding $\mathbf{U}_{i+n}$ is not the gradient of a function $\phi\left(v^{1}, v^{2}, \ldots v^{n}\right)$.

Let us now consider a set of Newton equations (2.1) admitting a Lagrangian $L\left(\mathbf{q}^{i}, \dot{\mathbf{q}}^{i}, t\right)$ (or many $s$-equivalent Lagrangians $L_{\rho}$ ). Its action $S_{L}$ can be put in a form invariant under a reparametrization, by enlarging the configuration space from $\left\{\mathbf{q}^{i}\right\}$ to $\left\{\mathbf{q}^{i}(\tau), t(\tau)\right\}$

$$
S_{L}=\int d \tau \dot{t}(\tau) L\left(\mathbf{q}^{i}(\tau), \frac{\dot{\mathbf{q}}^{i}(\tau)}{i(\tau)}, t(\tau)\right)
$$

In the $n$-time approach, a canonical structure $\Gamma_{a b}$, satisfying Eq. (3.76), corresponds to $L$, and, therefore, there is a set of first-class constraints (5.2) in the enlarged phase space $\bar{M}$. The associated Dirac Hamiltonian $H_{D}$ [see Eq. (5.30) ], gives a set of Hamilton equations, where $\mathbf{x}^{i}(\tau)$ $=\mathbf{x}^{i}\left(t^{1}(\tau), t^{2}(\tau), \ldots, t^{n}(\tau)\right)$. The first half of these equations, implemented with the constraints $(5.2)$, is 


$$
\begin{aligned}
& t^{i}(\tau)=-\lambda^{i}(\tau), \\
& \dot{\mathbf{x}}^{i}(\tau)=\sum_{j} \lambda^{j}(\tau)\left\{\mathbf{x}^{i}(\tau), \psi_{j}\right\}^{\prime}, \\
& \psi_{i}=\epsilon_{i}-H_{i}(\mathbf{x}, \mathbf{p}, t)=0 .
\end{aligned}
$$

When these equations can be inverted to get $\epsilon_{i}, \mathbf{p}_{i}, \lambda^{i}$ in terms of $\mathbf{x}^{i}, \dot{\mathbf{x}}^{i}, t^{i}, \dot{t}^{i}$, the following inverse Legendre transformation generates a singular Lagrangian $L_{1}$, depending on $\mathbf{x}^{i}$ and not on $\mathbf{q}^{i}$

$$
L_{1}\left(\mathbf{x}^{i}(\tau), t^{i}(\tau), \dot{\mathbf{x}}^{i}(\tau)\right)=\mathbf{p}_{i} \cdot \dot{\mathbf{x}}^{i}-\epsilon_{i} \dot{t}^{i}-H_{D} .
$$

Its Hessian has $n$ null eigenvectors, implying the existence of the $n$ first-class constraints (5.2), and of $n$ gauge invariances of $L_{1}$, of which one is the $\tau$-reparametrization invariance, which guarantees $H_{c}=0$.

The $L_{1}$ corresponding to the case of a harmonic oscillator has been calculated in Ref. 12, and it is given in Appendix A. It turns out that

$$
S_{1}=\int d \tau L_{1} \neq \int\left(\mathbf{f}^{i} \cdot d \mathbf{x}^{i}+g_{i} d t^{i}\right)
$$

(that is, it is associated to a generalization of Finsler geome$\operatorname{try}^{50}$ ), showing once again that a predictive action does not exist. However, as shown in Ref. 12, $S_{1}$ restricted at equal times reproduces Eq. (5.54), since at equal times it is $q^{i}=\mathbf{x}^{i}$. (See Ref. 51 for other forms of the no-interaction theorem in the relativistic case, based on singular Lagrangians.)

Let us remark that the systems we are considering have constraints $\psi_{i}$ linear in the $\epsilon_{i}$. Therefore, the first two of Eqs. (5.55) do not depend on $\epsilon_{i}$, and the $\lambda^{i}$ are always equal to the $-\dot{t}^{i}$. This fact prevents the use of the so-called Dirac Lagrangian $L_{D}$, often used for the path integral of the relativistic systems, whose $\psi_{i}$ are at least quadratics in the $\epsilon_{i}=p_{i}^{0}$

$$
L_{D}\left(x^{i, \mu}, \dot{x}^{i, \mu}, \lambda^{i}\right)=p_{i, \mu} \dot{x}^{i, \mu}-H_{D},
$$

where the $p_{i, \mu}$ are obtained from the first half of the Hamilton equations [they are analogous to the first two of Eq. (5.55) ], but the equations $\psi_{i}=0$ are not required. In $L_{D}$ the $\lambda^{i}$ are Lagrange multipliers, which are considered as einbeins $^{52}$ to recover the constraints $\psi_{i}=0$ (they appear as secondary constraints from the primary ones

$$
\left.\pi_{i}=\frac{\partial L_{D}}{\partial \dot{\lambda}^{i}}=0\right) .
$$

For instance, for the free relativistic particle in the massless limit, the corresponding equations (5.55) cannot be solved for the $\lambda^{i}$, and only $L_{D}$ is available.

When the Newton equations (2.1) do not admit a Lagrangian $L$, there are still the first-class constraints for each allowed canonical structure $\Gamma_{a b}$, not necessarily satisfying Eqs. (3.76). In this case, either $L_{1}$ does not exist [Eqs. (5.55) are not invertible in the $\left.\mathbf{p}_{i}\right]$, or $L_{1}$ does not admit an equal time limit.

\section{CONCLUSIONS}

To conclude let us summarize the results obtained. Starting from the Newton equations (2.1), the $n$-time second-order equations (2.6) were obtained, and then they were transformed in the first-order system (2.9). To each solution $U_{\alpha}^{0}$ of Eqs. (3.44) [modulo a canonical transformation of the kind of Eq. (3.40)] a symplectic structure $\left(\Gamma^{-1}\right)^{a b}$ is associated. To each of these symplectic structures there is an associated set of first-class constraints (5.2) in the enlarged phase space $\bar{M}$. We have also seen how to recover the original equations (2.9) from each canonical structure, by means of the Droz-Vincent equations (5.30) and (5.31).

Let us remark that what we have constructed are only local canonical structure $\left(\Gamma^{-1}\right)^{a b}$ : whether some or all of them can be globalized will depend on the given system; it is also possible that no global canonical structure will exist.

The open problem is which canonical structure is more relevant from the physical point of view; different Hamiltonians will generate different classical and quantum theories. (See Ref. 25 for a review of these ambiguities, and for a rich bibliography on the argument.)

When the Newton equations (2.1) admit one Lagrangian [modulo the trivial transformation $L \rightarrow L+d F(q, t)$ / $d t$, there is only one canonical structure with a $\Gamma$, which satisfies Eq. (3.76), thus allowing to recover the Lagrangian $L$. This is the preferred canonical structure, and at equal times we recover the standard Hamiltonian formalism, with $\mathbf{q}^{i}=\mathbf{x}^{i}$ and

$$
H=\sum_{i} H_{i} .
$$

When the Newton equations (2.1) admit $K$ (with possibly $K=\infty$ ) s-equivalent Lagrangians $L_{\rho}$, with $\rho=1,2, \ldots, K$, there are $K$ canonical structures with $\Gamma_{a b}^{(\rho)}$ satisfying Eqs. (3.76). Some of them will be excluded because of the lack of a canonical realization of the kinematical algebra (of the Galilei group, in this case) (see Ref. 53 for a noncanonical realization of the Lorentz algebra). Thus only say $L_{\rho^{\prime}}$ and $\Gamma_{a b}^{\left(\rho^{\prime}\right)}$, with $\rho^{\prime}=1,2, \ldots, h \leqslant K$, are left, and, as shown in Ref. 41 , each of them canonically realizes a different subgroup of the dynamical symmetries of the equations (2.1). When the system has no bound states, one selects the unique $\left.\Gamma_{a b}^{(} \rho^{\prime}\right)$, which satisfies the separability condition. ${ }^{54}$ The other restrictions which can be imposed are that: (1) $H=\Sigma_{i} H_{i}$ must be interpretable as the energy of the system; (2) if we add a perturbation, the perturbed Newton equations must still allow the existence of at least one Lagrangian ${ }^{55}$ : this is a very stringent condition, which usually singles out a unique $L$, and a unique canonical structure; (3) the equal time action $S_{\left(\rho^{\prime}\right)}=\int d t L_{\left(\rho^{\prime}\right)}$ becomes the phase of the wave function at the quantum level: therefore, at least in principle, an interference experiment could discriminate among the various $S_{\left(\rho^{\prime}\right)}{ }^{56}$ When the Newton equations (2.1) do not allow any Lagrangian, a canonical structure with $\Gamma_{a b}$ satisfying Eq. (3.76) does not exist. In general, even at equal times, we have $\left\{q^{i, m}, q^{j, k}\right\} \neq 0$, and, since we do not have a good definition of the energy, we cannot give any particular significance to $H=\Sigma_{i} H_{i}$. The other two restrictions are the requirement of a canonical realization of the kinematical group, and the requirement (3) above. Only if the dynamics is separable, a unique canonical structure is singled out.

Once a canonical structure has been chosen, we obtain a well defined set of first-class constraints associated to the 
original Newton equations, even when they are coupled to external fields. In general the coupling to external fields will be not minimal; in the constraint's approach this implies that, when there is a well defined one-time theory underlying the first class constraints, like in this case, not every coupling with external fields will be allowed, but only those which preserve the first-class character of the constraints. See for a comparison the restrictions on the external supersymmetric fields, when coupled to matter supermultiplets in Ref. 57.

Let us remark that the chosen canonical structure in the presymplectic manifold $M$ has to be identified with the class of Dirac brackets, which can be defined starting from the constraint's theory in the enlarged phase space $\bar{M}$, with the gauge fixings $t_{1}-t_{2}=$ const. Indeed, in our construction, there is the underlying hypothesis that the time variables are globally defined on $M$.

As already noticed the present approach can be applied to relativistic particle systems described by a set of first-class constraints. One has to solve the mass-shell constraints in the energies and to apply the present analysis to each determination of the energy spectrum. In this way the manifest covariance is lost and, at the quantum level, this would correspond to the not manifestly covariant Hamiltonian approach of Feshback-Villars ${ }^{58}$ after the Foldy-Wouthuysen transformation has been performed. ${ }^{59}$

\section{APPENDIX A: AN EXAMPLE}

In order to give an explicit example of the procedure sketched in Sec. II, let us consider the very simple case of a two particles system, with a harmonic mutual force. The one-time equations of motion of this system are

$$
\begin{aligned}
& m_{1} \ddot{\mathbf{q}}^{1}=-k\left(\mathbf{q}^{1}-\mathbf{q}^{2}\right), \\
& m_{2} \ddot{\mathbf{q}}^{2}=+k\left(\mathbf{q}^{1}-\mathbf{q}^{2}\right),
\end{aligned}
$$

where $k$ is the elastic constant. In this simple case it is possible to get the following explicit expression of the two-time forces:

$$
\begin{aligned}
\mathscr{F}^{1}= & +\left(m_{1} \omega^{2} / \Delta\right)\left\{-\omega(1+\alpha \cos (\omega \tau))\left(\mathbf{q}^{1}-\mathbf{q}^{2}\right)\right. \\
& +\alpha(\omega \tau \cos (\omega \tau)-\sin (\omega \tau)) \mathbf{v}^{1} \\
& \left.+(\omega \tau+\alpha \sin (\omega \tau)) \mathbf{v}^{2}\right\}, \\
\mathscr{F}^{2}= & -\alpha\left(m_{2} \omega^{2} / \Delta\right)\left\{-\omega(\alpha+\cos (\omega \tau))\left(\mathbf{q}^{1}-\mathbf{q}^{2}\right)\right. \\
& +(\alpha \omega \tau+\sin (\omega \tau)) \mathbf{v}^{1} \\
& \left.+(\omega \tau \cos (\omega \tau)-\sin (\omega \tau)) \mathbf{v}^{2}\right\},
\end{aligned}
$$

where

$$
\begin{aligned}
& \alpha=m_{1} / m_{2}, \quad \omega=\sqrt{k / \mu}, \\
& \mu=m_{1} m_{2} / m, \quad m=m_{1}+m_{2},
\end{aligned}
$$

and

$$
\begin{aligned}
& \tau=t^{1}-t^{2}, \\
& \Delta=\omega\left[1+\alpha^{2}+2 \alpha \cos (\omega \tau)+\alpha \omega \tau \sin (\omega \tau)\right] .
\end{aligned}
$$

It is easily verified that, when $\tau=0$, we get the forces (A1). Moreover, we may verify that the integrability conditions

$$
\frac{d \mathscr{F}^{1}}{d t^{2}}=0, \frac{d \mathscr{F}^{2}}{d t^{1}}=0
$$

are satisfied.

In Eqs. (A2) the $\mathrm{q}^{i}$ and the $\mathrm{v}^{i}(i=1,2)$ must be understood as functions of their own time $t^{i}$ :

$$
\mathbf{q}^{1}=\mathbf{q}^{1}\left(t^{1}\right), \quad \mathbf{q}^{2}=\mathbf{q}^{2}\left(t^{2}\right),
$$

and so forth.

The general solution of the two-time equations of motion

$$
m_{i} \mathbf{a}^{i}\left(t^{i}\right)=\mathscr{F}^{i}\left(\tau, \mathbf{q}^{1}-\mathbf{q}^{2}, \mathbf{v}^{i}\right),
$$

can be written

$$
\begin{aligned}
& \mathbf{q}^{1}\left(t^{1}\right)=\mathbf{a}+\mathbf{b} t^{1}+\mathbf{c} \cos \left(\omega t^{1}\right)+\mathbf{d} \sin \left(\omega t^{1}\right), \\
& \mathbf{q}^{2}\left(t^{2}\right)=\mathbf{a}+\mathbf{b} t^{2}-\alpha\left[\mathbf{c} \cos \left(\omega t^{2}\right)+\mathbf{d} \sin \left(\omega t^{2}\right)\right],
\end{aligned}
$$

where $\mathbf{a}, \mathbf{b}, \mathbf{c}$, and $\mathbf{d}$ are 12 constraints of integration.

Observe that $\mathscr{F}^{1}+\mathscr{F}^{2}$ is in general different from zero, except when $\tau=0$. That is, the action-reaction law is satisfied at $\tau=0$ only.

Also observe that, since the original set of equations (A1) is autonomous, the forces (A2) depend on $\tau=t^{1}-t^{2}$ only.

We give here without demonstrations an example of two first class constraints, describing the dynamics of two particles of masses $m_{1}$ and $m_{2}$, respectively,

$$
\psi_{i}=\epsilon_{i}-H_{i} \approx 0 \quad(i=1,2),
$$

with

$$
H_{i}=\left(1 / 2 m_{i}\right)\left[\mathbf{p}_{i}{ }^{2}+V\left(\rho^{2}\right)\right],
$$

where

$$
\rho=\mathbf{r}-(\tau / m) \mathbf{p},
$$

and

$$
\mathbf{p}=\mathbf{p}_{1}+\mathbf{p}_{2}, \quad \mathbf{r}=\mathbf{x}^{1}-\mathbf{x}^{2} .
$$

Here $\mathbf{x}^{i}$ are the canonical variables for the two particles. When we consider the equal time dynamics, we have to add the two constraints and to put $t^{1}=t^{2}$. In this case we have $\mathbf{x}^{i}=\mathbf{q}^{i}$, and the sum of the two constraints becomes the usual conservation of the total energy, with a potential given by

$$
(1 / 2 \mu) V\left(\mathbf{r}^{2}\right) \text {, }
$$

$\mu$ being the reduced mass.

In the case of a harmonic oscillator we have to choose

$$
V\left(\rho^{2}\right)=\mu^{2} \omega^{2} \rho^{2} \text {. }
$$

In this last case it is possible to give the explicit expression of the singular Lagrangian, mentioned in Sec. V, and given in Ref. 12. Its expression is the following:

$L_{1}=\left[1+\kappa \frac{m_{1} m_{2}}{\dot{t}^{1} \dot{t}^{2}}\left(\frac{\dot{t}^{1}}{m_{1}}-\frac{\dot{t}^{2}}{m^{2}}\right)^{2}\right]^{-1} \cdot\left[\frac{m_{1}\left(v^{1}\right)^{2}}{2 \dot{t}^{1}}+\frac{m_{2}\left(v^{2}\right)^{2}}{2 \dot{t}^{2}}+\kappa \frac{m_{1} m_{2}}{\dot{t}^{1} \dot{t}^{2}}\left(\frac{\dot{t}^{1}}{m_{1}}+\frac{\dot{t}^{2}}{m_{2}}\right)\left(v^{1}-v^{2}\right)^{2}\right]-\frac{\mu^{2} \omega^{2}}{2}\left(\frac{\dot{t}^{1}}{m_{1}}+\frac{\dot{t}^{2}}{m_{2}}\right) \mathbf{r}^{2}$, 
where

$$
\kappa=\mu^{2} \omega^{2} \tau^{2} / m^{2},
$$

and

$$
v^{i}=\dot{\mathbf{x}}^{i}+\frac{\mu^{2} \omega^{2} \tau}{m}\left(\frac{\dot{t}^{1}}{m_{1}}+\frac{\dot{t}^{2}}{m_{2}}\right) \mathbf{r} .
$$

\section{APPENDIX B: DISCUSSION OF EQ. (3.44)}

In this appendix we want to show that the equations for $U_{a}$ and $V_{i}$

$$
\begin{aligned}
& \Gamma_{a b} h_{i}^{b}=\Gamma_{a i}, \\
& \Gamma_{a b} h_{i}^{a} h_{j}^{b}=\Gamma_{i j}
\end{aligned}
$$

(where we recall that $a, b=1,2, \ldots, 2 N$; and $i, j=1,2, \ldots, n$ ) imply for the $\lambda_{i}$ defined by the equation

$$
V_{i}=U_{a} h_{i}^{a}+\lambda_{i}
$$

the condition

$$
Y_{j} \lambda_{i}=Y_{i} \lambda_{j}
$$

which has for general solution

$$
\lambda_{i}=-Y_{i} \Phi,
$$

with $\Phi$ an arbitrary function of $\left\{y^{\alpha}\right\}$ and $\left\{t^{i}\right\}$.

If we perform the substitution (3.46) in the expression for the $\Gamma_{a i}$, and use Eqs. (3.18'), we get

$$
\begin{aligned}
\Gamma_{a i}= & \frac{\partial U_{a}}{\partial t^{i}}+\frac{\partial}{\partial y^{a}}\left(U_{b} h_{i}^{b}+\lambda_{i}\right) \\
= & \frac{\partial U_{a}}{\partial t^{i}}-\Gamma_{a b} h_{i}^{b}+h_{i}^{b} \frac{\partial U_{a}}{\partial y^{b}} \\
& +U_{b} \frac{\partial h_{i}^{b}}{\partial y^{a}}+\frac{\partial \lambda_{i}}{\partial y^{a}} \\
= & Y_{i} U_{a}+U_{b} \frac{\partial h_{i}^{b}}{\partial y^{a}}+\frac{\partial \lambda_{i}}{\partial y^{a}}+\Gamma_{a i},
\end{aligned}
$$

from which we get the following equation for the $\left\{U_{a}\right\}$, once the $\lambda_{i}$ are given

$$
Y_{i} U_{a}=-U_{b} \frac{\partial h_{i}^{b}}{\partial y^{a}}-\frac{\partial \lambda_{i}}{\partial y^{a}} .
$$

The second of Eqs. (3.18') can be written

$$
\begin{aligned}
& h_{i}^{a} Y_{j} U_{a}-h_{j}^{a} Y_{i} U_{a} \\
& \quad=-U_{a}\left(\frac{\partial h_{i}^{a}}{\partial t^{j}}-\frac{\partial h_{j}^{a}}{\partial t^{i}}\right)-\left(\frac{\partial \lambda_{i}}{\partial t^{j}}-\frac{\partial \lambda_{j}}{\partial t^{i}}\right),
\end{aligned}
$$

which, using Eq. (B1), gives

$$
Y_{j} \lambda_{i}-Y_{i} \lambda_{j}+U_{a}\left(Y_{j} h_{i}^{a}-Y_{i} h_{j}^{a}\right)=0 .
$$

But the $h_{i}^{a}$ satisfy the integrability conditions (3.8), so we get

$$
Y_{j} \lambda_{i}=Y_{i} \lambda_{j}
$$

The general solution of these equations in $\lambda_{i}$ is given by Eq. (3.48), since they are the integrability conditions in order that Eq. (3.48) could be integrated in $\Phi$, for given $\lambda_{i}$.

With Eq. (3.48) the functions $V_{i}$ can be written

$$
V_{i}=U_{a} h_{i}^{a}-Y_{i} \Phi \text {, }
$$

so that Eq. (B1) for the $U_{a}$ becomes

$$
Y_{i} U_{a}+U_{b} \frac{\partial h_{i}^{b}}{\partial y^{a}}=Y_{i} \frac{\partial \Phi}{\partial y^{a}}+\frac{\partial \Phi}{\partial y^{b}} \frac{\partial h_{i}^{b}}{\partial y^{a}},
$$

since

$$
\left[\frac{\partial}{\partial y^{a}}, Y_{i}\right]=\frac{\partial h_{i}^{b}}{\partial y^{a}} \frac{\partial}{\partial y^{b}},
$$

or

$$
Y_{i}\left(U_{a}-\frac{\partial \Phi}{\partial y^{a}}\right)+\frac{\partial h_{i}^{b}}{\partial y^{a}}\left(U_{b}-\frac{\partial \Phi}{\partial y^{b}}\right)=0,
$$

which is Eq. (3.44). So we finally have

$$
U_{a}=U_{a}^{0}+\frac{\partial \Phi}{\partial y^{a}},
$$

and

$$
V_{i}=U_{a}^{0} h_{i}^{a}-\frac{\partial \Phi}{\partial t^{i}},
$$

with $U_{a}^{0}$ satisfying Eq. (3.44), which is the wanted result.

It is now easily verified that Eq. (3.44) for the $U_{a}^{0}$ are integrable. Indeed the integrability conditions of a system like

$$
Y_{i} U_{a}=A_{a i}(y, t, U),
$$

are

$$
Y_{j} A_{a i}=Y_{i} A_{a j},
$$

as it is easily verified using Eq. (3.25).

For the Eq. (3.44) the condition (B5) becomes

$$
Y_{j}\left(U_{b}^{o} \frac{\partial h_{i}^{b}}{\partial y^{a}}\right)=(i \leftrightarrow j),
$$

that is

$$
U_{b}^{0} Y_{j} \frac{\partial h_{i}^{b}}{\partial y^{a}}-U_{c}^{0} \frac{\partial h_{j}^{c}}{\partial y^{b}} \frac{\partial h_{i}^{b}}{\partial y^{a}}=(i \leftrightarrow j),
$$

or, using Eq. (B2)

$U_{b}^{o} \frac{\partial}{\partial y^{a}} Y_{j} h_{i}^{b}-U_{c}^{o}\left(\frac{\partial h_{j}^{c}}{\partial y^{b}} \frac{\partial h_{i}^{b}}{\partial y^{a}}+\frac{\partial h_{j}^{b}}{\partial y^{a}} \frac{\partial h_{i}^{c}}{\partial y^{b}}\right)=(i \leftrightarrow j)$, and finally

$$
U_{b}^{0} \frac{\partial}{\partial y^{a}}\left(Y_{j} h_{i}^{b}-Y_{i} h_{j}^{b}\right)=0,
$$

which is satisfied. So the equation for $U_{a}^{0}$ are integrable.

Another way to get the result (3.48) and the last result, or better to see how to integrate the equations for $U_{a}^{0}$, once the general solution of the original equations of motion is known, is the following.

Let us introduce the new variables

$$
z^{a}=\tilde{z}^{a}(y, t),
$$

defined as the solutions of the system

$$
Y_{i} \tilde{z}^{a}=\left(\frac{\partial}{\partial t^{i}}+h_{i}^{a} \frac{\partial}{\partial y^{a}}\right) \tilde{z}^{a}=0 .
$$

This system is completely integrable, since the vector fields $Y_{i}$ are commuting. It follows that it has $2 N$ independent solutions, which we call $\tilde{z}^{a}(y, t)$. For a general function $f(y, t)$ let us put 
$f(\boldsymbol{y}, t)=\tilde{f}(z, t)$.

From Eq. (B8) we have

$$
Y_{i} f(y, t)=\frac{\partial}{\partial t^{i}} \tilde{f}(z, t),
$$

and this means that Eqs. (3.47) can be written

$$
\frac{\partial}{\partial t^{j}} \tilde{\lambda}_{i}(z, t)=\frac{\partial}{\partial t^{i}} \tilde{\lambda}_{j}(z, t),
$$

which has the general solution

$$
\tilde{\lambda}_{i}(z, t)=-\frac{\partial}{\partial t^{i}} \widetilde{\Phi}(z, t)=-Y_{i} \Phi(y, t) .
$$

Transforming the equation for the $U_{a}^{0}$ to the new variables, that is Eq. (3.44), we get

$$
\frac{\partial}{\partial t^{i}} \widetilde{U}_{a}^{o}(z, t)=-\widetilde{U}_{b}^{0}(z, t) \frac{\partial \tilde{z}^{c}}{\partial y^{a}} \frac{\partial}{\partial z^{c}}\left(\frac{\partial \tilde{y}^{b}}{\partial t^{i}}\right),
$$

where $\tilde{y}^{b}(z, t)$ are the old variables in terms of the new ones. Since

$$
\frac{\partial \tilde{z}^{a}}{\partial y^{b}} \frac{\partial \tilde{y}^{b}}{\partial z^{c}}=\delta_{c}^{a},
$$

for the required independence of the $\tilde{z}^{a}$, we get

$$
\frac{\partial \tilde{y}^{a}}{\partial z^{c}} \frac{\partial \widetilde{U}_{a}^{0}}{\partial t^{i}}=-\widetilde{U}_{b}^{0} \frac{\partial}{\partial t^{i}} \frac{\partial \tilde{y}^{b}}{\partial z^{c}},
$$

or

$$
\frac{\partial}{\partial t^{i}}\left(\frac{\partial \tilde{y}^{a}}{\partial z^{b}} \widetilde{U}_{a}^{0}\right)=0 \text {. }
$$

These equations have the general solution for $\widetilde{U}_{a}^{0}$

$$
\widetilde{U}_{a}^{0}=K_{a}(\tilde{z}(y, t)),
$$

where the functions $K_{a}$ are $2 N$ arbitrary functions.

Clearly, the variables $z^{a}$ are nothing more than the complete set of the constants of motion of the original system of equations of motion. Their knowledge determines the $U_{a}^{0}$, with the arbitrariness expressed by Eq. (B14), and in turn they determine the whole class of solutions for the $U_{a}$ and $V_{i}$.

The one-form $\theta^{0}$ of Eq. (3.50) can now be written

$$
\theta^{o}=K_{a} \frac{\partial \tilde{y}^{a}}{\partial z^{b}} d z^{b}+K_{a}\left(\frac{\partial \tilde{y}^{a}}{\partial t^{i}}-\tilde{h}_{i}^{a}\right) d t^{i}
$$

but, from the definition of the variables $z^{a}=\tilde{z}^{a}(y, t)$, and from their assumed invertibility, we get

$$
\frac{\partial \tilde{z}^{a}}{\partial y^{b}} \frac{\partial \tilde{y}^{b}}{\partial t^{i}}+\frac{\partial \tilde{z}^{a}}{\partial t^{i}}=0
$$

which, beside Eq. (B8), tells us that

$$
\frac{\partial \tilde{y}^{b}}{\partial t^{i}}=\tilde{h}_{i}^{b} \text {. }
$$

So we get

$\theta^{0}=K_{a} \frac{\partial \tilde{y}^{a}}{\partial z^{b}} d z^{b}$

Clearly, we may choose

$$
\begin{aligned}
& K_{a} \frac{\partial \tilde{y}^{a}}{\partial z^{b}}=z^{b+N}, \quad \text { for } b=1,2, \ldots, N, \\
& K_{a} \frac{\partial \tilde{y}^{a}}{\partial z^{b}}=0, \text { for } b=N+1, N+2, \ldots, 2 N,
\end{aligned}
$$

which is a solution which satisfies the condition (3.17). Indeed, we may write $\Gamma_{a b}$ in the following way:

$$
\Gamma_{a b}=\frac{\partial \tilde{z}^{c}}{\partial y^{a}} W_{c d} \frac{\partial \tilde{z}^{d}}{\partial y^{b}}
$$

with

$$
W_{c d}=\frac{\partial}{\partial z^{d}}\left(\frac{\partial \tilde{y}^{a}}{\partial z^{c}} \widetilde{U}_{a}^{0}\right)-\frac{\partial}{\partial z^{c}}\left(\frac{\partial \tilde{y}^{a}}{\partial z^{d}} \widetilde{U}_{a}^{0}\right),
$$

and it is only necessary to verify that $W_{c d}$ is nonsingular. But with the choice (B19), we see that $W_{c d}$ is the symplectic metric:

$$
W_{c d}=\delta_{c+N, d}-\delta_{d+N, c},
$$

which has determinant equal to +1 .

With the choice (B19) we have

$$
\theta^{0}=\sum_{b=1}^{N} z^{b+N} d z^{b},
$$

which is in symplectic form, and shows the connection of the variables $\bar{p}_{i}$ and $\bar{x}^{i}(i=1,2, \ldots, N)$, with the constants of motion $z^{a}(a=1,2, \ldots, 2 N)$ [see Eq. (3.53) and (3.49)].

${ }^{1}$ E. E. Salpeter and H. Bethe, Phys. Rev. 84, 1232 (1951); M. Gell-Mann and F. Low, ibid. 84, 350 (1951); G. C. Wick, ibid. 96, 1124 (1954); M. Nakanishi, Suppl. Prog. Theor. Phys. 43, 1 (1969); A. A. Arkhipov and I. V. Savrin, Serpukhov report No. 83-50, 1983 (unpublished).

${ }^{2}$ I. T. Todorov, Report No. Comm. JINRE2-10125, Dubna, 1976 (unpublished); Ann. Inst. H. Poincaré A 28, 207 (1978).

${ }^{3}$ A. Komar, Phys. Rev. D 18, 1881, 1887, 3617 (1978); 19, 2908 (1979).

${ }^{4}$ (a) Theory of Action-at-a-Distance in Relativistic Particle Mechanics, edited by E. H. Kerner (Gordon and Breach, New York, 1972); (b) Relativistic Action-at-a-Distance: Classical and Quantum Aspects, edited by J. A. Llosa, Lectures Notes in Physics, Vol. 162 (Springer, Berlin, 1982); (c) Constraint's Theory and Relativistic Dynamics, Firenze, 1986, edited by G. Longhi and L. Lusanna (World Scientific, Singapore, 1987); (d) E. C. G. Sudarshan and N. Mukunda, Classical Mechanics: A Modern Perspective (Wiley, New York, 1974).

${ }^{5}$ H. W. Crater and P. Van Alstine, Phys. Rev. D 30, 2585 ( 1984); H. Sazdjian, ibid. 33, 3401, 3425, 3435 (1986); Phys. Lett. B 156, 381 (1985).

${ }^{6}$ P. A. M. Dirac, Can. J. Math. 2, 129 (1950); Lectures on Quantum Mechanics, Belfer Graduate School of Science, Monographs Series, Yeshiva University, New York, 1964; J. L. Anderson and P. G. Bergmann, Phys. Rev. 83, 1018 (1951); P. G. Bergmann and J. Goldberg, ibid. 98, 531 (1955); E. C. G. Sudarshan and N. Mukunda, Classical Mechanics: A Modern Perspective, (Wiley, New York, 1974); A. Lichnerowicz, C. R. Acad. Sci. Paris A 280, 523 (1975); A. J. Hanson, T. Regge, and C. Teitelboim, Constrained Hamiltonian Systems, Contributi del Centro Linceo Interdisciplinare di Scienze Matematiche, Fisiche e loro Applicazioni, no. 22, Accademia Nazionale dei Lincei, Roma, 1975; K. Sundermeyer, Constrained Dynamics with Applications to Yang-Mills Theory, General Relativity, Classical Spin, Dual String Model, Lecture Notes in Physics, Vol. 169 (Springer, Berlin, 1982).

${ }^{7}$ Ph. Droz-Vincent, Lett. Nuovo Cimento 1, 839 (1969); Phys. Scr. 2, 129 (1970); Nuovo Cimento B 12, 1 (1972); Ann. Inst. H. Poincaré A 20, 269 (1974); A 27, 407 (1977); A 32, 377 (1980); Rep. Math. Phys. 8, 79 (1975).

${ }^{8}$ Ph. Droz-Vincent, Lett. Nuovo Cimento 1, 800 (1971); Phys. Rev. D 19, 702 (1979); Nuovo Cimento A 58, 355 (1980); Lett. Nuovo Cimento, 30, 375 (1981); 33, 383 (1982); 35, 83 (1982); 38, 177 (1983); 40, 165 (1984); 44, 199 (1985); Phys. Rev. D 29, 687 (1984). 
${ }^{9}$ V. A. Rizov, H. Sazdjian, and I. T. Todorov, Ann. Phys. (NY) 165, 59 (1985).

${ }^{10}$ V. Iranzo, J. Llosa, F. Marqués, and A. Molina, Ann. Inst. H. Poincaré, A 40, 1 (1984).

${ }^{11}$ G. Longhi and L. Lusanna, Phys. Rev. D 34, 3707 (1986).

${ }^{12}$ L. Lusanna, in Proceedings of the VII Seminaron Problems of High Energy Physics and Quantum field Theory, (Protvino, USSR, 1984), Vol. I, p. 123; in Proceedings of the IVM. Grossmann Meeting, Rome, 1985, edited by R. Ruffini (Elsevier, Amsterdam, 1986), p. 1163.

${ }^{13} \mathrm{Ph}$. Droz-Vincent, Lett. Nuovo Cimento 23, 184 (1978); 28, 166 (1980); in Ref. 4(b) and 4(c); J. Geom. Phys. 2, 101 (1985)

${ }^{14}$ M. H. L. Pryce, Proc. R. Soc. London A 195, 62 (1948); T. D Newton and E. P. Wigner, Rev. Mod. Phys. 21, 400 (1949); L. L. Foldy and S. A. Wouthuysen, Phys. Rev. 78, 29 (1950); T. F. Jordan and N. Mukunda, ibid. 132, 1842 (1953); E. H. Kerner, J. Math. Phys. 6, 1218 (1965); A. J. Kalnay, "The Localization Problem" in Studies in Foundations, Methodology and Philosophy of Science, edited by M. Bunge (Springer, Berlin, 1971), Vol. 4; M. Lorente and P. Roman, J. Math. Phys. 15, 70 (1974); J. Niederle, "Localizability of Particles" in Hadron Constituents and Symmetries, Smolenice, 1976, edited by J. Lonik and M. Noga (Veda, Bratislavia, 1978), Vol. 3; H. Sazdjian, Nucl. Phys. B 161, 469 (1979).

${ }^{15}$ I. T. Todorov, Sissa report, Trieste, 1980 (unpublished); in Quantum Theory, Groups, Fields and Particles, edited by A. O. Barut (Reidel, Dordrecht, 1983); T. Takabayasi, Suppl. Prog. Theor. Phys. 67, 1 (1979); L. Lusanna, Nuovo Cimento B 65, 135 (1981); G. Longhi, in Ref. 4(b).

${ }^{16}$ L. Bel, Mecanica Relativista Predictiva, Report UAB FT-34 de la Universidad Autonoma de Barcelona, 1977 (unpublished); in Differential Geometry and Relativity, edited by M. Cahen and M. Flato (Reidel, Dordrecht, 1976), p. 197; R. N. Hill, in Ref. 4(b); V. Iranzo, J. A. Llosa, and A. Molina, Ann. Phys. (NY) 160, 114 (1983); J. M. Pons, ibid. 148, 192 (1983); D. G. Currie and T. F. Jordan, in Quantum Theory and Statistical Physics, Boulder, 1967, edited by A. O. Barut and W. E. Brittin (Gordon and Breach, New York, 1968), Vol. XA, p. 91.

${ }^{17}$ L. Bel, Ann. Inst. H. Poincaré A 12, 307 (1970); 14, 189 (1971); 18, 57 (1973), and in Ref. 4(b); R. Arens, Arch. Rat. Mech. Anal. 47, 255 (1972), and in Ref. 4(b); L. Bel and J. Martin, Ann. Inst. H. Poincaré A 22, 173 (1975); 33, 409 (1980); 34, 231 (1981); D. Hirondel, J. Math. Phys. 15, 1689 (1974); 20, 104 (1979); L. Bel and X. Fustero, Ann. Inst. H. Poincaré A 25, 411 (1976).

${ }^{18}$ D. G. Currie, T. F. Jordan, and E. C. G. Sudarshan, Rev. Mod. Phys. 35, 350 (1963); H. Leutwyler, Nuovo Cimento 37, 556 (1965); E. Kerner, J. Math. Phys. 6, 1218 (1965); L. Bel. Ann. Inst. H. Poincaré A 14, 189 (1971); Ph. Droz-Vincent, Nuovo Cimento 12, 1 (1972); Rep. Math. Phys. 8, 79 (1975); A. F. Kracklauer, J. Math. Phys. 17, 693 (1976); A. Salas, ibid. 20, 2459 (1979); V. Iranzo, J. Llosa, F. Marqués, and A. Molina, ibid. 24, 1665 (1983); F. Marqués, V. Iranzo, A. Molina, A. Montoto, and J. Llosa, Phys. Rev. D 31, 314 (1985); X. Jaen, A. Molina, and V. Iranzo, J. Math. Phys. 27, 512 (1986); J. Pons, Ann. Phys. (NY) 148, 192 (1983). For a review, see S. Chelkowski, J. Nietendel, and R. Suchanek, Acta Phys. Polon. B 11, 809 (1980).

${ }^{19}$ P. A. M. Dirac, Rev. Mod. Phys. 21, 392 (1949).

${ }^{20}$ Ph. Droz-Vincent, Rep. Math. Phys. 8, 79 (1975).

${ }^{21}$ E. T. Whittaker, A Treatise on the Analytical Dynamics of Particles and Rigid Bodies (Cambridge U. P., New York, 1960), p. 275.

${ }^{22}$ R. N. Hill, J. Math. Phys. 8, 1756 (1966).

${ }^{23}$ W. Sarlet and F. Cantrijn, Hadronic J. 1, 101, 1497 (1978)

${ }^{24}$ W. Sarlet, J. Phys. A 15, 1503 (1982).

${ }^{25}$ G. M. Morandi, C. Ferrario, G. Lo Vecchio, G. Marmo, and C. R. Rubano, "The inverse problem in the calculus of variations and the geometry of the tangent bundle," preprint Univ. Napoli, 1987.

${ }^{26}$ D. G. Currie, Phys. Rev. 142, 817 (1966); R. N. Hill, J. Math. Phys. 8, 201 (1967).

${ }^{27}$ M. J. Gotay, Proc. Am. Math. Soc. 84, 111 (1982)

${ }^{28}$ J. M. Souriau, Structure Des Systèmes Dynamiques (Dunod, Paris, 1970).
See also Ref. 39.

${ }^{29}$ A. Komar, Phys. Rev. D 18, 1881 (1978); 19, 2908 (1979); C. Piron, in Quantum Theory, Groups, Fields and Particles, edited by A. O. Barut (Reidel, Dordrecht, 1983).

${ }^{30} \mathrm{G}$. Longhi, "Multitime approach to nonrelativistic and relativistic quantum mechanics," in Actas de los Encuentros Relativistas '84, St. Ander, 1984, edited by the Universidad de Valencia, 1984, p. 131.

${ }^{31}$ G. Longhi, L. Lusanna, and J. M. Pons, in Ref. 4(c), p. 252.

${ }^{32}$ E. H. Kerner, J. Math. Phys. 6, 1218 (1965).

${ }^{33}$ See for instance L. P. Eisenhart, Continuous Groups of Transformations (Dover, New York, 1961).

${ }^{34}$ R. Abraham and J. E. Mardsen, Foundations of Mechanics, second ed. (Benjamin/Cummings, London, 1978).

${ }^{35}$ R. M. Santilli, Foundations of Theoretical Mechanics I, the Inverse Problem in Newtonian Mechanics (Springer, New York, 1978).

${ }^{36}$ W. Sarlet, Hadronic J. 2, 407 (1979); 3, 765 (1980); J. Phys. A 14, 2227 (1981); W. Sarlet and F. Cantrijn, Hadronic J. 1, 101 (1978).

${ }^{37}$ M. Crampin, J. Phys. A 14, 2567 (1981); M. Henneaux, ibid. 15, L93 (1982); Ann. Phys. (NY) 140, 45 (1982); P. De Ritis, G. Marmo, G. Platania, and P. Scudellaro, Int. J. Theor. Phys. 22, 931 (1983).

${ }^{38}$ M. Crampin, G. E. Prince, and G. Thomson, J. Phys. A 17, 1437 (1984).

${ }^{39} \mathrm{~N}$. Woodhouse, Geometric Quantization (Clarendon, Oxford, 1980).

${ }^{40}$ W. Sarlet and F. Cantrijn, SIAM Rev. 23, 467 (1981).

${ }^{4}$ 'N. Mukunda, A. P. Balachandran, Jan S. Nilsson, E. C. G. Sudarshan, and F. Zaccaria, Phys. Rev. D 23, 2189 ( 1981 ).

${ }^{42}$ See for instance Ref. 36.

${ }^{43}$ G. Marmo, E. J. Saletan, A. Simoni, and B. Vitale, Dynamical Systems (Wiley, Chichester, 1985).

${ }^{44}$ G. Marmo, G. Morandi, A. Simoni, and E. C. G. Sudarshan, "Quasiinvariance and central extensions,"preprint Univ. Napoli, 1986; G. Marmo, E. J. Saletan, and A. Simoni, Nuovo Cimento B 96, 159 (1986); see also E. C. G. Sudarshan and N. Mukunda, Classical Dynamics: A Modern Perspective (Wiley, New York, 1974).

${ }^{45}$ Y. Choquet-Bruhat, C. de Witt-Morette, and M. Dillard-Bleick, Analysis, Manifolds, and Physics (North-Holland, Amsterdam, 1977), p. 228.

${ }^{46}$ See for instance P. G. Bergmann and J. Goldberg, Phys. Rev. 98, 531 (1955); P. G. Bergmann, Rev. Mod. Phys. 33, 510 (1961).

${ }^{47}$ X. Jaen, J. Llosa, F. Marqués, and A. Montoto, J. Math. Phys. 27, 519 (1986).

${ }^{48}$ M. Henneaux, Ann. Phys. (NY) 140, 45 (1982).

${ }^{49}$ E. H. Kerner, J. Math. Phys. 3, 35 (1962); 6, 1218 (1965); R. P. Gaida, Yu. B. Klyuchkovskii, and V. I. Tretyak, Theor. Math. Phys. 44, 194 (1980); 45, 180 (1980); in Proceedings of the VII Seminar on Problems of High Energy Physics and Quantum Field Theory (Protvino, USSR, 1984); Vol. I, p. 99; in Proceedings of the Workshop on Constraint's Theory and Relativistic Dynamics, Firenze, 1986, edited by G. Longhi and L. Lusanna (World Scientific, Singapore, 1987), p. 210.

${ }^{50} \mathrm{H}$. Rund, The Differential Geometry of Finsler Spaces (Springer, Berlin, 1959).

${ }^{51}$ R. Giachetti and E. Sorace, Lett. Nuovo Cimento 26, 1 (1979); A. Barducci, R. Giachetti, and E. Sorace, Lett. Math. Phys. 8, 105 (1984); G. Marmo, N. Mukunda, and E. C. G. Sudarshan, Phys. Rev. D 30, 2110 (1984); A. P. Balachandran, G. Marmo, and A. Stern, Nuovo Cimento A 69, 175 (1982); J. Martin and J. L. Sanz, J. Math. Phys. 19, 780 (1978).

${ }^{52}$ L. Brink, S. Deser, B. Zumino, P. di Vecchia, and P. Hove, Phys. Lett. B 64, 435 (1976).

${ }^{53}$ T. F. Jordan, Phys. Rev. 166, 1308 (1968).

${ }^{54}$ R. N. Hill and E. H. Kerner, Phys. Rev. Lett. 17, 1156 (1966).

${ }^{55}$ M. Henneaux and L. C. Shepley, J. Math. Phys. 23, 2101 (1982).

${ }^{56}$ V. V. Dodonov, V. I. Man'ko, and V. D. Skarzhinsky, Hadronic J. 4, 1734 (1981).

${ }^{57}$ L. Lusanna and B. Milewski, Nucl. Phys. B 247, 396 (1984).

${ }^{58}$ H. Feshback and F. Villars, Rev. Mod. Phys. 30, 24 (1984).

${ }^{59}$ L. L. Foldy and S. A. Wouthuysen, Phys. Rev. 78, 29 (1950). 